\title{
A radiative model for the weak scale and neutrino mass via dark matter
}

\author{
Amine Ahriche, ${ }^{a, b}$ Kristian L.McDonald ${ }^{c}$ and Salah Nasri ${ }^{d}$ \\ ${ }^{a}$ Department of Physics, University of Jijel, \\ PB 98 Ouled Aissa, DZ-18000 Jijel, Algeria \\ ${ }^{b}$ The Abdus Salam International Centre for Theoretical Physics, \\ Strada Costiera 11, I-34014, Trieste, Italy \\ ${ }^{c}$ ARC Centre of Excellence for Particle Physics at the Terascale, \\ School of Physics, The University of Sydney, \\ NSW 2006, Australia \\ ${ }^{d}$ Physics Department, UAE University, \\ POB 17551, Al Ain, United Arab Emirates
}

E-mail: aahriche@ictp.it, klmcd@physics.usyd.edu.au, snasri@uaeu.ac.ae

ABSTRACT: We present a three-loop model of neutrino mass in which both the weak scale and neutrino mass arise as radiative effects. In this approach, the scales for electroweak symmetry breaking, dark matter, and the exotics responsible for neutrino mass, are related due to an underlying scale-invariance. This motivates the otherwise-independent $\mathcal{O}(\mathrm{TeV})$ exotic masses usually found in three-loop models of neutrino mass. We demonstrate the existence of viable parameter space and show that the model can be probed at colliders, precision experiments, and dark matter direct-detection experiments.

Keywords: Higgs Physics, Beyond Standard Model, Neutrino Physics

ARXIV EPRINT: 1508.02607 


\section{Contents}

1 Introduction 1

2 A scale-invariant three-loop model 3

2.1 Symmetry breaking 3

2.2 The scalar spectrum 5

3 Neutrino mass $\quad 6$

4 Experimental constraints $\quad 7$

4.1 Lepton flavor 7

4.2 Electroweak precision tests 8

4.3 Higgs invisible decay 8

4.4 The Higgs decay channel $h \rightarrow \gamma \gamma$

5 Dark matter $\quad 9$

5.1 Relic density 9

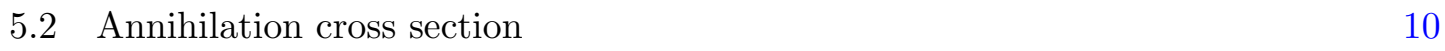

$\begin{array}{lll}5.3 & \text { Direct detection } & 12\end{array}$

$6 \quad$ Numerical analysis and results 12

$\begin{array}{lll}7 & \text { Conclusion } & 15\end{array}$

$\begin{array}{ll}\text { A Multi-scalar scale-invariant theories } & 16\end{array}$

$\begin{array}{lr}\text { B Oblique parameter functions } & 18\end{array}$

$\begin{array}{ll}\text { C Loop induced Higgs decay functions } & 19\end{array}$

\section{Introduction}

Radiative symmetry breaking [1] offers an interesting alternative to the conventional Higgs mechanism. In this approach, calculable weakly-coupled radiative effects induce symmetry breaking in classically scale-invariant theories, thereby giving birth to mass - a process known as dimensional transmutation. When applied to the Standard Model (SM), it is well known that radiative symmetry breaking is not viable, due to the destabilizing influence of the heavy top quark. However, the SM is known to be incomplete, due to e.g. an absence of massive neutrinos and the need to incorporate dark matter (DM). It is therefore interesting to consider the viability of radiative symmetry breaking within SM extensions. 
The addition of massive neutrinos and DM to the SM likely requires new degrees of freedom. When considering radiative symmetry breaking, there are a number of relevant considerations that can guide the choice of beyond-SM fields. The destabilizing radiative corrections from the top quark can be overcome by bosonic degrees of freedom with mass $\gtrsim 200 \mathrm{GeV}$. In principle these states could be much heavier than the $\mathrm{TeV}$ scale. However, radiative symmetry breaking typically introduces a single scale into a theory, with other mass and symmetry breaking scales related to this scale. ${ }^{1}$ Consequently both the electroweak scale and the mass scale for exotics may be related via dimensionless parameters. Thus, absent hierarchically small parameters [2], one anticipates exotics with $\mathcal{O}(\mathrm{TeV})$ masses.

In the LHC era, $\mathrm{TeV}$ scale exotics are of particular interest. However, efforts to generate tiny neutrino masses via weak-scale exotics can struggle to achieve the necessary mass-suppression, relative to the weak scale, without invoking tiny couplings. Perhaps the most obvious exception are models with radiative neutrino mass, as the inherent loopsuppression in such models can motivate lighter new physics. From this perspective, threeloop models of neutrino mass are particularly compelling, as the new physics is expected to be $\mathcal{O}(\mathrm{TeV})$.

These considerations focus our attention on scale-invariant models with three-loop neutrino mass. If we also seek to address the DM problem, a minimal approach would see the DM play a role in either generating neutrino mass or triggering electroweak symmetry breaking. Thus, we arrive at a picture in which both the weak scale and neutrino mass arise as radiative effects, with the weak scale, the DM mass, and the mass scale for the exotics that induce neutrino mass, all finding a common birth, via dimensional transmutation. This picture can address short-comings of the SM, while also explaining why the exotics required in three-loop neutrino mass models have (otherwise independent) masses of $\mathcal{O}(\mathrm{TeV})$ - a common ancestry requires that they be related to the weak scale.

In this work we present a scale-invariant model for three-loop neutrino mass that contains a fermionic DM candidate. We explore the model in detail and present feasible parameter space that achieves the correct DM relic abundance, while generating viable symmetry breaking and neutrino masses - all compatible with low-energy constraints. As per usual for scale-invariant frameworks, the model predicts a dilaton. However, here the dilaton has the dual role of allowing electroweak symmetry breaking and simultaneously sourcing the lepton number violation that allows radiative neutrino masses. We note that a number of earlier works studied relationships between the origin of neutrino mass and DM, see e.g. refs. [3-54]. There has also been much interest in scale-invariant models in recent years, see e.g. refs. [55-125].

The structure of this paper is as follows. In section 2 we introduce the model and detail the symmetry breaking sector. We turn our attention to the origin of neutrino mass in section 3 and discuss various constraints in section 4. Dark matter is discussed in section 5 and our main analysis and results appear in section 6. Conclusions are drawn in section 7.

\footnotetext{
${ }^{1}$ The exceptions being when a theory also contains a confining gauge sector, as with QCD in the SM, or a completely decoupled hidden sector possessing its own symmetry breaking and/or confining pattern.
} 


\section{A scale-invariant three-loop model}

We consider a classically scale-invariant (SI) extension of the SM in which neutrino mass appears at the three-loop level. The SM is extended by the addition of two charged scalars, $S_{1,2}^{+} \sim(1,1,2)$, three singlet fermions, $N_{i R} \sim(1,1,0)$, with $i \in\{1,2,3\}$ labeling generations, and a singlet scalar, $\phi \sim(1,1,0){ }^{2}$ A $Z_{2}$ symmetry with action $\left\{S_{2}, N_{R}\right\} \rightarrow$ $\left\{-S_{2},-N_{R}\right\}$ is imposed, with all other fields being $Z_{2}$-even. This symmetry remains exact in the full theory, making the lightest $Z_{2}$-odd field a stable DM candidate, which should be taken as the lightest fermion, $N_{1} \equiv N_{\mathrm{DM}}$, to avoid a cosmologically-excluded stable charged particle. The scalar $\phi$ plays a key role in triggering electroweak symmetry breaking, as explained below, and also ensures that lepton number symmetry is explicitly broken, thereby allowing radiative neutrino mass.

Consistent with the SI and $Z_{2}$ symmetries, the Lagrangian contains the following terms:

$$
\mathcal{L} \supset-\left\{f_{\alpha \beta} \overline{L_{\alpha}^{c}} L_{\beta} S_{1}^{+}+g_{i \alpha} \overline{N_{i}^{c}} S_{2}^{+} e_{\alpha R}+\text { H.c }\right\}-\frac{1}{2} \tilde{y}_{i} \phi \overline{N_{i}^{c}} N_{i}-V\left(H, S_{1,2}, \phi\right),
$$

where Greek letters label SM flavors, $\alpha, \beta \in\{e, \mu, \tau\}$, and $f_{\alpha \beta}, g_{i \alpha}$ and $\tilde{y}_{i}$ are Yukawa couplings. The $Z_{2}$ symmetry forbids the term $\bar{L} \tilde{H} N_{R}$, which would otherwise generate tree-level neutrino masses after the SM scalar $H \sim(1,2,1)$ develops a VEV. The potential $V\left(H, S_{1,2}, \phi\right)$ is the most-general potential consistent with the SI and $Z_{2}$ symmetries.

\section{$2.1 \quad$ Symmetry breaking}

We are interested in parameter space where both $\phi$ and $H$ acquire nonzero vacuum expectation values (VEVs), $\langle H\rangle \neq 0$ and $\langle\phi\rangle \neq 0$. This breaks both the SI and electroweak symmetries while preserving the $Z_{2}$ symmetry. The most-general scalar potential includes the terms

$$
\begin{aligned}
V_{0}\left(H, S_{1,2}, \phi\right) \supset & \lambda_{\mathrm{H}}|H|^{4}+\frac{\lambda_{\phi \mathrm{H}}}{2}|H|^{2} \phi^{2}+\frac{\lambda_{\phi}}{4} \phi^{4}+\frac{\lambda_{\mathrm{S}}}{4}\left(S_{1}^{-}\right)^{2}\left(S_{2}^{+}\right)^{2} \\
& +\sum_{a=1,2} \frac{1}{2}\left(\lambda_{\mathrm{H} a}|H|^{2}+\lambda_{\phi a} \phi^{2}\right)\left|S_{a}\right|^{2} .
\end{aligned}
$$

A complete analysis of the potential requires the inclusion of the leading-order radiative corrections. In general the full one-loop corrected potential is not analytically tractable. However, a useful approach for approximating the ground state in SI models was presented in ref. [126]. Taking guidance from ref. [126], we adopt an approximation for the ground state that allows one to obtain simple analytic expressions. The physical spectrum contains two charged scalars $S_{1,2}^{+}$, and two neutral scalars, denoted as $h_{1,2}$. As discussed in appendix A, for the present model, the minimum of the loop-corrected potential can be approximated by neglecting loop corrections involving only the scalars $h_{1,2}$. The viability of this simplification follows from the dominance of the beyond-SM scalars $S_{1,2}^{+}$

\footnotetext{
${ }^{2}$ Quantities in parentheses refer to quantum numbers under the SM gauge symmetry $\mathrm{SU}(3)_{c} \otimes \mathrm{SU}(2)_{L} \otimes$ $\mathrm{U}(1)_{Y}$.
} 
(see appendix A). Adopting this approximation, the one-loop corrected potential for the CP-even neutral scalars is

$$
\begin{aligned}
V_{1-l}(h, \phi) & =\frac{\lambda_{\mathrm{H}}}{4} h^{4}+\frac{\lambda_{\phi \mathrm{H}}}{4} \phi^{2} h^{2}+\frac{\lambda_{\phi}}{4} \phi^{4}+\sum_{i=\text { all fields }} n_{i} G\left(m_{i}^{2}(h, \phi)\right), \\
G(\eta) & =\frac{\eta^{2}}{64 \pi^{2}}\left[\log \frac{\eta}{\Lambda^{2}}-\frac{3}{2}\right],
\end{aligned}
$$

where $\Lambda$ is the renormalization scale, $n_{i}$ are the field multiplicities, and we employ the unitary gauge, with $H=(0, h / \sqrt{2})^{T}$. The sum is over all fields, neglecting the light SM fermions (all but the top quark) and the (to be determined) neutral scalar mass-eigenstates $h_{1,2}$. Due to the SI symmetry, the field-dependent masses can be written as

$$
m_{i}^{2}(h, \phi)=\frac{\alpha_{i}}{2} h^{2}+\frac{\beta_{i}}{2} \phi^{2},
$$

the constants $\alpha_{i}$ and $\beta_{i}$ are given by

$$
\begin{array}{llll}
\alpha_{W}=\frac{g^{2}}{2}, \quad \alpha_{Z}=\frac{g^{2}+g^{\prime 2}}{2}, & \alpha_{t}=y_{t}^{2}, & \alpha_{S_{a}}=\lambda_{\mathrm{H} a}, & \alpha_{N_{i}}=0, \\
\beta_{W}=\beta_{Z}=\beta_{t}=0, & \beta_{S_{a}}=\lambda_{\phi a}, & \beta_{N_{i}}=2 \tilde{y}_{i}^{2},
\end{array}
$$

with $g\left(g^{\prime}\right)$ and $y_{t}$ are the $\mathrm{SU}(2)_{L}\left(\mathrm{U}(1)_{Y}\right)$ gauge and top Yukawa couplings, respectively.

Dimensional transmutation introduces a dimensionful parameter into the theory in exchange for one of the dimensionless couplings. In the present model, an analysis of the potential shows that a minimum with $\langle h\rangle \equiv v \neq 0$ and $\langle\phi\rangle \equiv x \neq 0$ exists for $\lambda_{\phi \mathrm{H}}<0$, and is triggered at the scale where the couplings satisfy the relation

$$
2\left\{\lambda_{\mathrm{H}} \lambda_{\phi}+\frac{\lambda_{\mathrm{H}}}{x^{2}} \sum_{i} n_{i}\left\{\beta_{i}-\alpha_{i} \frac{v^{2}}{x^{2}}\right\} G^{\prime}\left(m_{i}^{2}\right)\right\}^{1 / 2}+\lambda_{\phi \mathrm{H}}+\frac{2}{x^{2}} \sum_{i} n_{i} \alpha_{i} G^{\prime}\left(m_{i}^{2}\right)=0,
$$

with $G^{\prime}(\eta)=\partial G(\eta) / \partial \eta$. The further condition

$$
-\frac{\lambda_{\phi \mathrm{H}}}{2 \lambda_{\mathrm{H}}}=\frac{v^{2}}{x^{2}}+\sum_{i} \frac{n_{i} \alpha_{i}}{\lambda_{\mathrm{H}} x^{2}} G^{\prime}\left(m_{i}^{2}\right)
$$

is also satisfied at the minimum. Thus, for $\lambda_{\phi \mathrm{H}, \mathrm{H}}=\mathcal{O}(1)$ one has $v \sim x$ and the exotic scale is naively expected around the TeV scale. Note that eqs. (2.7) and (2.8) ensure that the tadpoles vanish. ${ }^{3}$

Defining the one-loop quartic couplings as

$$
\lambda_{\phi}^{1-l}=\frac{1}{6} \frac{\partial^{4} V_{1-l}}{\partial \phi^{4}}, \quad \lambda_{\mathrm{H}}^{1-l}=\frac{1}{6} \frac{\partial^{4} V_{1-l}}{\partial h^{4}}, \quad \lambda_{\phi \mathrm{H}}^{1-l}=\frac{\partial^{4} V_{1-l}}{\partial h^{2} \partial \phi^{2}},
$$

vacuum stability at one-loop requires that the following conditions be satisfied:

$$
\lambda_{\mathrm{H}}^{1-l}, \lambda_{\phi}^{1-l}, \lambda_{\phi \mathrm{H}}^{1-l}+2 \sqrt{\lambda_{\mathrm{H}}^{1-l} \lambda_{\phi}^{1-l}}>0 .
$$

\footnotetext{
${ }^{3}$ To our level of approximation, eqs. (2.7) and (2.8) are the loop-corrected generalizations of the standard tree-level results, $4 \sqrt{\lambda_{\mathrm{H}}(\Lambda) \lambda_{\phi}(\Lambda)}+\lambda_{\phi \mathrm{H}}(\Lambda)=0$ and $\lambda_{\phi \mathrm{H}} / 2 \lambda_{\mathrm{H}}=v^{2} / x^{2}[127]$.
} 
We must also impose the condition $\lambda_{\phi \mathrm{H}}^{1-l}<0$ to ensure that the vacuum with $v \neq 0$ and $x \neq 0$ is the ground state. ${ }^{4}$ Eq. (2.10) also guarantees that the eigenmasses-squared for the CP-even neutral scalars are strictly positive, and forces one of the beyond-SM scalars $S_{1,2}^{+}$be the heaviest particle in the spectrum.

\subsection{The scalar spectrum}

The mass matrix for the neutral scalars is denoted as

$$
V_{1-l}(h, \phi) \supset \frac{1}{2}(h, \phi)\left(\begin{array}{cc}
m_{h h}^{2} & m_{h \phi}^{2} \\
m_{h \phi}^{2} & m_{\phi \phi}^{2}
\end{array}\right)\left(\begin{array}{l}
h \\
\phi
\end{array}\right),
$$

where the mass parameters $m_{h h}, m_{\phi \phi}$ and $m_{h \phi}$ are calculated from the loop-corrected potential $V_{1-l}(h, \phi)$. The mass eigenstates are labeled as

$$
h_{1}=\cos \theta_{h} h-\sin \theta_{h} \phi, \quad h_{2}=\sin \theta_{h} h+\cos \theta_{h} \phi,
$$

with the eigenvalues and mixing angles given by

$$
\begin{aligned}
M_{h_{1,2}}^{2} & =\frac{1}{2}\left\{m_{11}^{2}+m_{22}^{2} \pm \sqrt{\left(m_{22}^{2}-m_{11}^{2}\right)^{2}+4 m_{12}^{4}}\right\}, \\
\tan 2 \theta_{h} & =\frac{2 m_{12}^{2}}{m_{22}^{2}-m_{11}^{2}} .
\end{aligned}
$$

Here $h_{1}$ is a massive SM-like scalar and $h_{2}$ is a pseudo-Goldstone boson associated with SI symmetry breaking - the latter is massless at tree-level but acquires mass at the loop-level. One can obtain simple tree-level expressions for the SM-like scalar mass

$$
M_{h_{1}}^{2}=\left(2 \lambda_{\mathrm{H}}-\lambda_{\phi \mathrm{H}}\right) v^{2},
$$

and the mixing angle,

$$
c_{h} \equiv \cos \theta_{h}=\frac{x}{\sqrt{x^{2}+v^{2}}}, \quad s_{h} \equiv \sin \theta_{h}=\frac{v}{\sqrt{x^{2}+v^{2}}},
$$

though in large regions of parameter space it is important to include loop corrections to these expressions to obtain accurate results. In our numerical analysis we employ the full loop-corrected expressions for the scalar masses and mixing, as is necessary to obtain $M_{h_{2}} \neq 0$. Due to the SI symmetry, the parameters in the model are somewhat constrained, with $\lambda_{\phi}$ and $\lambda_{\phi \mathrm{H}}$ fixed by eqs. (2.7) and (2.8) while the Higgs mass $M_{h_{1}} \simeq 125 \mathrm{GeV}$ fixes $\lambda_{\mathrm{H}}$.

The tree-level masses for the charged scalars, $S_{1,2}^{+}$, are

$$
M_{S_{a}}^{2}=\frac{1}{2}\left\{\lambda_{\phi a} x^{2}+\lambda_{\mathrm{H} a} v^{2}\right\} \quad \text { for } \quad a=1,2,
$$

where $S_{1}^{+}$and $S_{2}^{+}$do not mix due to the $Z_{2}$ symmetry. Note that a useful approximation for $M_{h_{2}}$ is [126]

$$
M_{h_{2}}^{2} \simeq \frac{1}{8 \pi^{2}\left(\langle\phi\rangle^{2}+\langle h\rangle^{2}\right)}\left\{M_{h_{1}}^{4}+6 M_{W}^{4}+3 M_{Z}^{4}-12 M_{t}^{4}+2 \sum_{a=1}^{2} M_{S_{a}}^{4}-2 \sum_{i=1}^{3} M_{N_{i}}^{4}\right\},
$$

\footnotetext{
${ }^{4}$ For $\lambda_{\phi \mathrm{H}}^{1-l}>0$ the vacuum with only one nonzero VEV is preferred.
} 


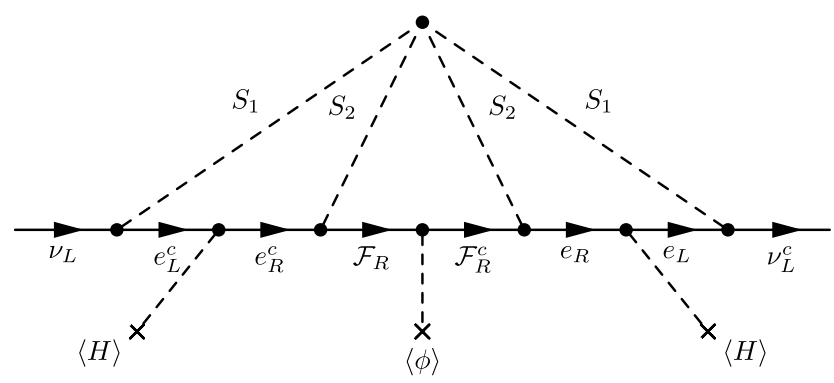

Figure 1. Three-loop diagram for neutrino mass in a scale-invariant model.

which shows that one of the beyond-SM scalars $S_{1,2}$ must be the heaviest beyond-SM state in order to ensure $M_{h_{2}}>0$.

As mentioned already, we expect the VEVs to be of a similar scale, $\langle\phi\rangle \sim\langle h\rangle$, as evidenced by eq. (2.8). For completeness, however, we note that there is a technically natural limit in which one obtains $\langle\phi\rangle \gg\langle h\rangle$. This arises when all the couplings to $\phi$ are taken to be hierarchically small, namely $\left\{\tilde{y}_{i}, \lambda_{\phi \mathrm{H}}, \lambda_{\phi 1,2}\right\} \ll 1$, with the masses $M_{h_{1}}, M_{N}$ and $M_{S_{1,2}}$ held at $\mathcal{O}(\mathrm{TeV})$. This feature reflects the fact that $\phi$ decouples in the limit $\left\{\tilde{y}_{i}, \lambda_{\phi \mathrm{H}}, \lambda_{\phi 1,2}\right\} \rightarrow 0$, up to gravitational effects [128]. In this limit we expect the model to be very similar to the KNT model [3], but with a light, very weakly-coupled scalar in the spectrum, $h_{2}$. Absent a compelling motivation for such hierarchically small parameters, we restrict our attention to values of $\langle\phi\rangle \leq 5 \mathrm{TeV}$.

\section{$3 \quad$ Neutrino mass}

We now turn to the origin of neutrino mass. The $Z_{2}$-odd fermions, $N_{i}$, develop masses $M_{N_{i}}=\tilde{y}_{i}\langle\phi\rangle$, and do not mix with SM leptons due to the $Z_{2}$ symmetry. We order their masses as $M_{\mathrm{DM}} \equiv M_{N_{1}}<M_{N_{2}}<M_{N_{3}}$. SM neutrinos, on the other hand, acquire mass radiatively. The combination of the Yukawa interactions in eq. (2.1) and the term

$$
V\left(H, S_{1,2}, \phi\right) \supset \frac{\lambda_{\mathrm{S}}}{4}\left(S_{1}^{-}\right)^{2}\left(S_{2}^{+}\right)^{2},
$$

in the scalar potential, explicitly break lepton number symmetry. Consequently neutrino masses appear at the three-loop level as shown in figure 1 .

Calculating the loop diagram, the mass matrix has the form

$$
\left(\mathcal{M}_{\nu}\right)_{\alpha \beta}=\frac{\lambda_{\mathrm{S}}}{\left(4 \pi^{2}\right)^{3}} \frac{m_{\sigma} m_{\rho}}{M_{S_{2}}} g_{\sigma i}^{*} g_{\rho i}^{*} f_{\alpha \sigma} f_{\beta \rho} \times F_{\text {loop }}\left(\frac{M_{N_{i}}^{2}}{M_{S_{2}}^{2}}, \frac{M_{S_{1}}^{2}}{M_{S_{2}}^{2}}\right),
$$

where $m_{\sigma, \rho}$ denote charged lepton masses and the function $F_{\text {loop }}(x, y)$ encodes the loop integrals $[17-22]$

$$
F_{\text {loop }}(\alpha, \beta)=\frac{\sqrt{\alpha}}{8 \beta^{2}} \int_{0}^{\infty} d r \frac{r}{r+\alpha}\left(\int_{0}^{1} d x \ln \frac{x(1-x) r+(1-x) \beta+x}{x(1-x) r+x}\right)^{2} .
$$


One can relate the neutrino mass matrix to the elements of the Pontecorvo-MakiNakawaga-Sakata (PMNS) mixing matrix $[129,130]$ elements, we parameterize the latter as

$$
U_{\nu}=\left(\begin{array}{ccc}
c_{12} c_{13} & c_{13} s_{12} & s_{13} e^{-i \delta_{d}} \\
-c_{23} s_{12}-c_{12} s_{13} s_{23} e^{i \delta_{d}} & c_{12} c_{23}-s_{12} s_{13} s_{23} e^{i \delta_{d}} & c_{13} s_{23} \\
s_{12} s_{23}-c_{12} c_{23} s_{13} e^{i \delta_{d}} & -c_{12} s_{23}-c_{23} s_{12} s_{13} e^{i \delta_{d}} & c_{13} c_{23}
\end{array}\right) \times U_{m}
$$

with $\delta_{d}$ the Dirac phase and $U_{m}=\operatorname{diag}\left(1, e^{i \theta_{\alpha} / 2}, e^{i \theta_{\beta} / 2}\right)$ encoding the Majorana phase dependence. The shorthand $s_{i j} \equiv \sin \theta_{i j}$ and $c_{i j} \equiv \cos \theta_{i j}$ refers to the mixing angles. For our numerical scans (discussed below) we fit to the best-fit experimental values for the mixing angles and mass-squared differences: $s_{12}^{2}=0.320_{-0.017}^{+0.016}, s_{23}^{2}=0.43_{-0.03}^{+0.03}, s_{13}^{2}=0.025_{-0.003}^{+0.003}$, $\left|\Delta m_{13}^{2}\right|=2.55_{-0.09}^{+0.06} \times 10^{-3} \mathrm{eV}^{2}$ and $\Delta m_{21}^{2}=7.62_{-0.19}^{+0.19} \times 10^{-5} \mathrm{eV}^{2}$ [131]. Furthermore, we require that the contribution to neutrino-less double beta decay in this model satisfies the current bound. Within these ranges, one determines the parameter space where viable neutrino masses and mixing occur in the model.

\section{Experimental constraints}

In this section we discuss the constraints on the model from the lepton flavor violating process $\mu \rightarrow e \gamma$, the electroweak precision tests, the invisible Higgs decay, and the effect on $h \gamma \gamma$ process.

\subsection{Lepton flavor}

Flavor changing processes like $\mu \rightarrow e+\gamma$ arise via loop diagrams containing virtual charged scalars and give important constraints on the model. At one-loop the branching ratio for $\mu \rightarrow e+\gamma$ is

$$
\begin{aligned}
\mathcal{B}(\mu \rightarrow e \gamma) & =\frac{\Gamma(\mu \rightarrow e+\gamma)}{\Gamma(\mu \rightarrow e+\nu+\bar{\nu})} \\
& \simeq \frac{\alpha v^{4}}{384 \pi} \times\left\{\frac{\left|f_{\mu \tau} f_{\tau e}^{*}\right|^{2}}{M_{S_{1}}^{4}}+\frac{36}{M_{S_{2}}^{4}}\left|\sum_{i} g_{i e}^{*} g_{i \mu} F_{2}\left(M_{i}^{2} / M_{S_{2}}^{2}\right)\right|^{2}\right\}
\end{aligned}
$$

where $F_{2}(R)=\left[1-6 R+3 R^{2}+2 R^{3}-6 R^{2} \log R\right] /\left[6(1-R)^{4}\right]$. The corresponding expression for $\mathcal{B} \tau \rightarrow \mu+\gamma$ ) follows from a simple change of flavor labels in eq. (4.1). Similarly, the one-loop contributions to the anomalous magnetic moment of the muon are

$$
\delta a_{\mu}=-\frac{m_{\mu}^{2}}{16 \pi^{2}}\left\{\sum_{\alpha \neq \mu} \frac{\left|f_{\mu \alpha}\right|^{2}}{6 M_{S_{1}}^{2}}+\sum_{i} \frac{\left|g_{i \mu}\right|^{2}}{M_{S_{2}}^{2}} F_{2}\left(M_{i}^{2} / M_{S_{2}}^{2}\right)\right\} .
$$

Null-results from searches for neutrino-less double-beta decay give an additional constraint of $\left(\mathcal{M}_{\nu}\right)_{e e} \lesssim 0.35 \mathrm{eV}$ [132], though we find this is easily satisfied. 


\subsection{Electroweak precision tests}

In principle, precision electroweak measurements can provide additional constraints. The oblique parameters characterizing new physics effects are given by [133-138]

$$
\begin{aligned}
\frac{\alpha}{4 s_{W}^{2} c_{W}^{2}} S & =\frac{A_{Z Z}\left(M_{Z}^{2}\right)-A_{Z Z}(0)}{M_{Z}^{2}}-\left.\frac{\partial A_{\gamma \gamma}\left(q^{2}\right)}{\partial q^{2}}\right|_{q^{2}=0}+\left.\frac{c_{W}^{2}-s_{W}^{2}}{c_{W} s_{W}} \frac{\partial A_{\gamma Z}\left(q^{2}\right)}{\partial q^{2}}\right|_{q^{2}=0}, \\
\alpha T & =\frac{A_{W W}(0)}{M_{W}^{2}}-\frac{A_{Z Z}(0)}{M_{Z}^{2}} .
\end{aligned}
$$

Here, $\alpha=e^{2} /(4 \pi)=g^{2} s_{\mathrm{w}}^{2} /(4 \pi)$ is the fine-structure constant, $s_{\mathrm{w}}=\sin \theta_{\mathrm{w}}$ and $c_{\mathrm{w}}=\cos \theta_{\mathrm{w}}$ are the sine and cosine, respectively, of the Weinberg angle $\theta_{\mathrm{w}}$, and the functions $A_{V V^{\prime}}\left(q^{2}\right)$ are the coefficients of $g^{\mu \nu}$ in the vacuum-polarization tensors $\Pi_{V V^{\prime}}^{\mu \nu}(q)=g^{\mu \nu} A_{V V^{\prime}}\left(q^{2}\right)+$ $q^{\mu} q^{\nu} B_{V V^{\prime}}\left(q^{2}\right)$, where $V V^{\prime}$ could be either $\gamma \gamma, \gamma Z, Z Z$, or $W W$. In our model, the oblique parameters are given by [139]

$$
\begin{aligned}
\Delta T= & \frac{3}{16 \pi s_{\mathrm{w}}^{2} M_{W}^{2}}\left\{c_{h}^{2}\left[F\left(M_{Z}^{2}, M_{h_{1}}^{2}\right)-F\left(M_{W}^{2}, M_{h_{1}}^{2}\right)\right]+s_{h}^{2}\left[F\left(M_{Z}^{2}, M_{h_{2}}^{2}\right)-F\left(M_{W}^{2}, M_{h_{2}}^{2}\right)\right]\right. \\
& \left.-\left[F\left(M_{Z}^{2}, M_{h}^{2}\right)-F\left(M_{W}^{2}, M_{h}^{2}\right)\right]\right\}, \\
\Delta S= & \frac{1}{24 \pi}\left\{4 s_{\mathrm{w}}^{4} G\left(M_{S_{1}}^{2}, M_{S_{1}}^{2}, M_{Z}^{2}\right)+4 s_{\mathrm{w}}^{4} G\left(M_{S_{2}}^{2}, M_{S_{2}}^{2}, M_{Z}^{2}\right)+c_{h}^{2} \ln \frac{M_{h_{1}}^{2}}{M_{h}^{2}}+s_{h}^{2} \ln \frac{M_{h_{2}}^{2}}{M_{h}^{2}}\right. \\
& \left.+c_{h}^{2} \hat{G}\left(M_{h_{1}}^{2}, M_{Z}^{2}\right)+s_{h}^{2} \hat{G}\left(M_{h_{2}}^{2}, M_{Z}^{2}\right)-\hat{G}\left(M_{h}^{2}, M_{Z}^{2}\right)\right\},
\end{aligned}
$$

where the functions $F, G$ and $\hat{G}$ are given in the appendix and $M_{h}=125.09 \mathrm{GeV}$ denotes the reference value.

\subsection{Higgs invisible decay}

The model can also face constraints from the invisible Higgs decay, $\mathcal{B}(h \rightarrow \operatorname{inv})<17 \%$ [140]. In our case we have inv $\equiv\left\{h_{2} h_{2}\right\},\left\{N_{\text {DM }} N_{\text {DM }}\right\}$, when kinematically available. The corresponding decay widths are given by

$$
\begin{aligned}
\Gamma\left(h_{1} \rightarrow h_{2} h_{2}\right) & =\frac{1}{32 \pi} \frac{\left(\lambda_{122}\right)^{2}}{M_{h_{1}}}\left(1-\frac{4 M_{h_{2}}^{2}}{M_{h_{1}}^{2}}\right)^{\frac{1}{2}} \Theta\left(M_{h_{1}}-2 M_{h_{2}}\right), \\
\Gamma\left(h_{1} \rightarrow N_{\mathrm{DM}} N_{\mathrm{DM}}\right) & =\frac{\tilde{y}_{\mathrm{DM}}^{2} s_{h}^{2}}{16 \pi} M_{h_{1}}\left(1-\frac{4 M_{\mathrm{DM}}^{2}}{M_{h_{1}}^{2}}\right)^{\frac{3}{2}} \Theta\left(M_{h_{1}}-2 M_{\mathrm{DM}}\right) .
\end{aligned}
$$

The effective cubic coupling $\lambda_{122}$ is defined below in eq. (5.8). Due to the SI symmetry, we find that $\lambda_{122}$ vanishes at tree-level, with the small (loop-level) coupling sufficient to ensure the decay to $h_{2}$ pairs is highly suppressed. ${ }^{5}$

\footnotetext{
${ }^{5}$ Note that $h_{2}$ decays to SM states, much like a light SM Higgs boson but with suppression from the mixing angle, $s_{h}^{2}$. However, currently there are no dedicated ATLAS or CMS searches for light scalars in the channels $2 b, 2 \tau$ or $2 \gamma$, so we classify the decay $h_{1} \rightarrow h_{2} h_{2}$ as invisible. In practice the suppression of $\Gamma\left(h_{1} \rightarrow h_{2} h_{2}\right)$ due to SI symmetry renders this point moot.
} 


\subsection{The Higgs decay channel $h \rightarrow \gamma \gamma$}

The existence of extra charged scalars modifies the two Higgs branching ratios $\mathcal{B}(h \rightarrow$ $\gamma \gamma, \gamma Z$ ), and this deviation can be parameterized by the ratios:

$$
\begin{aligned}
& R_{\gamma \gamma}=\frac{\mathcal{B}(h \rightarrow \gamma \gamma)}{\mathcal{B}^{\mathrm{SM}}(h \rightarrow \gamma \gamma)}=\left|1+\frac{v}{2 c_{h}} \frac{\frac{\vartheta_{1}}{m_{S_{1}}^{2}} A_{0}^{\gamma \gamma}\left(\tau_{S_{1}}\right)+\frac{\vartheta_{2}}{m_{S_{2}}^{2}} A_{0}^{\gamma \gamma}\left(\tau_{S_{2}}\right)}{A_{1}^{\gamma \gamma}\left(\tau_{W}\right)+N_{c} Q_{t}^{2} A_{1 / 2}^{\gamma \gamma}\left(\tau_{t}\right)}\right|^{2}, \\
& R_{\gamma Z}=\frac{\mathcal{B}(h \rightarrow \gamma Z)}{\mathcal{B}^{\mathrm{SM}}(h \rightarrow \gamma Z)}=\left|1+\frac{s_{\mathrm{w}} v}{c_{h}} \frac{\frac{\vartheta_{1}}{m_{S_{1}}^{2}} A_{0}^{\gamma Z}\left(\tau_{S_{1}}, \lambda_{S_{1}}\right)+\frac{\vartheta_{2}}{m_{S_{2}}^{2}} A_{0}^{\gamma Z}\left(\tau_{S_{2}}, \lambda_{S_{2}}\right)}{c_{\mathrm{w}} A_{1}^{\gamma Z}\left(\tau_{W}, \lambda_{W}\right)+\frac{2\left(1-8 s_{\mathrm{w}}^{2} / 3\right)}{c_{\mathrm{w}}} A_{1 / 2}^{\gamma Z}\left(\tau_{t}, \lambda_{t}\right)}\right|^{2},
\end{aligned}
$$

where $\tau_{X}=M_{h_{1}}^{2} / 4 M_{X}^{2}$ and $\lambda_{X}=M_{Z}^{2} / 4 M_{X}^{2}$, with $M_{X}$ is the mass of the charged particle $X$ running in the loop, $N_{c}=3$ is the color number, $Q_{t}$ is the electric charge of the top quark in unit of $|e|$, and the loop amplitudes $A_{i}$ for spin 0 , spin $1 / 2$ and spin 1 particle contribution [141], which are given in the appendix. Here $\vartheta_{i}$, are the SM-like Higgs couplings to the pairs of charged scalars $S_{1,2}^{ \pm}$, which are given by

$$
\vartheta_{a}=c_{h} \lambda_{\mathrm{H} a} v+s_{h} \lambda_{\phi a} x
$$

The effect of the charged scalars on (4.8) and (4.9) depends on the masses for $S_{a}^{ \pm}$, the sign and the strength of their couplings to the SM Higgs doublet and the neutral singlet and on the mixing angle $\theta_{h}$. One can use the reported results from LHC to constraints these parameters.

\section{Dark matter}

\subsection{Relic density}

The lightest $Z_{2}$-odd field is a stable DM candidate. As mentioned already, the lightest exotic fermion $N_{\mathrm{DM}} \equiv N_{1}$ is the only viable DM candidate in the model. The relic density is given by [142-144]

$$
\Omega_{\mathrm{DM}} h^{2}=\frac{1.04 \times 10^{9} \mathrm{GeV}^{-1}}{M_{\mathrm{Pl}}} \frac{1}{\sqrt{g_{*}\left(T_{f}\right)}<\sigma v_{r}\left(x_{f}\right)>},
$$

where $M_{\mathrm{Pl}}=1.22 \times 10^{19} \mathrm{GeV}$ is the Planck scale, $g_{*}(T)$ is the total effective number of relativistic particle at temperature $T$, and

$$
\begin{aligned}
&\left\langle\sigma\left(N_{\mathrm{DM}} N_{\mathrm{DM}}\right) v_{r}\right\rangle= \sum_{X}\left\langle\sigma\left(N_{\mathrm{DM}} N_{\mathrm{DM}} \rightarrow X\right) v_{r}\right\rangle=\frac{1}{8 T M_{\mathrm{DM}}^{4} K_{2}^{2}\left(\frac{M_{\mathrm{DM}}}{T}\right)} \times \\
& \int_{4 M_{\mathrm{DM}}^{2}}^{\infty} d s \sigma_{N_{\mathrm{DM}}} N_{\mathrm{DM}} \rightarrow \text { all } \\
&
\end{aligned}
$$

is the thermally averaged DM annihilation cross-section, $v_{r}$ is the relative velocity, $s$ is the Mandelstam variable, $K_{1,2}$ are the modified Bessel functions and $\sigma_{N_{\mathrm{DM}} N_{\mathrm{DM}} \rightarrow \text { all }}(s)$ is the annihilation cross into all kinematically accessible final state particles at the CM energy $\sqrt{s}$. 


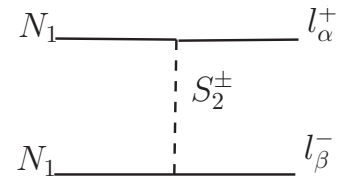

(a)

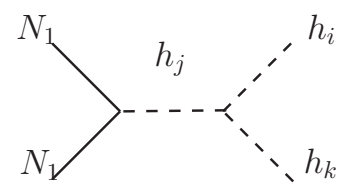

(d)

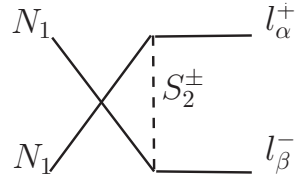

(b)

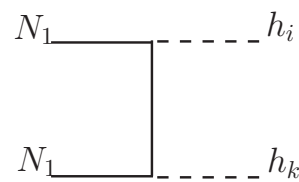

(e)

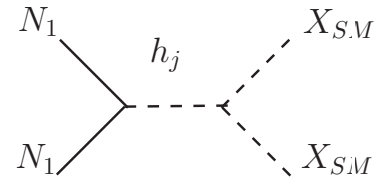

(c)

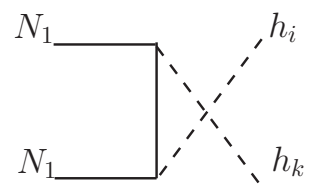

$(f)$

Figure 2. Different diagrams for DM annihilation.

The parameter $x_{f}=M_{\mathrm{DM}} / T_{f}$ represents the freeze-out temperature, and can be computed from

$$
x_{f}=\ln \frac{0.03 M_{\mathrm{Pl}} M_{\mathrm{DM}}<\sigma v_{r}\left(x_{f}\right)>}{\sqrt{T_{f}} x_{f}} .
$$

As will be discussed in the next section, we require that $\Omega_{N_{\mathrm{DM}}} h^{2}$ to be in agreement with the observed value of the dark matter relic density [145].

The thermally averaged annihilation cross-section can be approximated in the nonrelativistic limit as $\left\langle\sigma v_{r}\right\rangle=a+b v_{r}^{2}$, where $v_{r}$ is the relative DM velocity and $a$ and $b$ are the $s$-wave and $p$-wave factors, which receives contributions from different annihilation channels. In this limit, the velocity squared is approximated by $v_{r}^{2} \simeq 6 / x_{f}$. Here, we evaluate the thermally averaged cross section exactly following (5.2).

\subsection{Annihilation cross section}

In our model, there are many contributions, where the channels can be classified into three types according to their Feynman diagrams types: (1) annihilation into charged leptons $N_{\mathrm{DM}} N_{\mathrm{DM}} \rightarrow \ell_{\alpha}^{\mp} \ell_{\beta}^{ \pm}$(figure 2-a and -b), which are $t$-channel diagrams mediated by charged scalars ${ }^{6}(2)$ annihilation into SM fermions and gauge bosons pairs $N_{\mathrm{DM}} N_{\mathrm{DM}} \rightarrow$ $f \bar{f}, W^{-} W^{+}, Z Z$ (figure 2-c), which occur through s-channel $h_{1,2}$-mediated diagrams, and (3) the annihilations into scalars, $N_{\mathrm{DM}} N_{\mathrm{DM}} \rightarrow h_{1,2} h_{1,2}$ (figure 2-d, -e and -f), which occur through both $s$ - and $t$-channel diagrams.

Charged leptons annihilation channel. The DM $N_{1}$ couples to SM leptons through the Yukawa couplings $g_{1 \alpha}$, and can annihilate into charged lepton pairs as shown in figure 2 -a and $-b$. The cross section for annihilation into charged leptons ${ }^{7}$ is given by [146]

$$
\begin{aligned}
\sigma\left(N_{\mathrm{DM}} N_{\mathrm{DM}} \rightarrow \ell_{\alpha}^{-} \ell_{\beta}^{+}\right) v_{r}= & \frac{1}{8 \pi} \frac{\left|g_{1 \alpha} g_{1 \beta}^{*}\right|^{2}}{s\left(M_{S^{+}}^{2}-M_{\mathrm{DM}}^{2}+\frac{s}{2}\right)^{2}}\left[\frac{m_{\ell_{\alpha}}^{2}+m_{\ell_{\beta}}^{2}}{2}\left(\frac{s}{2}-M_{\mathrm{DM}}^{2}\right)\right. \\
& {\left[+\frac{8}{3} \frac{\left(M_{S^{+}}^{2}-M_{\mathrm{DM}}^{2}\right)^{2}+\frac{s}{2}\left(M_{S^{+}}^{2}-M_{\mathrm{DM}}^{2}\right)+\frac{s^{2}}{8}}{\left(M_{S^{+}}^{2}-M_{\mathrm{DM}}^{2}+\frac{s}{2}\right)^{2}}\left(\frac{s}{4}-M_{\mathrm{DM}}^{2}\right)\right], }
\end{aligned}
$$

\footnotetext{
${ }^{6}$ Actually, for the same flavor case there two $s$-channel diagrams mediated by $h_{1,2}$, however we neglect them due to the suppressed Higgs charged leptons couplings.

${ }^{7}$ Indeed for same flavor charged leptons $(\alpha=\beta)$, there are $h_{1,2}$ mediated $s$-channel processes that are proportional to their Yukawa couplings; we ignore these due to the Yukawa suppression.
} 
SM fermions and gauge boson channels. The processes $N_{\mathrm{DM}} N_{\mathrm{DM}} \rightarrow b \bar{b}, t \bar{t}, W^{+} W^{-}$ and $Z Z$ can occur as shown in figure 2-c. The corresponding amplitude can be written as

$$
\mathcal{M}=i c_{h} s_{h} y_{1} \bar{u}\left(k_{2}\right) u\left(k_{1}\right)\left(\frac{i}{s-M_{h_{1}}^{2}}-\frac{i}{s-M_{h_{2}}^{2}}\right) \mathcal{M}_{h \rightarrow S M}\left(m_{h} \rightarrow \sqrt{s}\right),
$$

where $\mathcal{M}_{h \rightarrow S M}\left(m_{h} \rightarrow \sqrt{s}\right)$ is the amplitude of the Higgs decay $h \rightarrow X_{\mathrm{SM}} \bar{X}_{\mathrm{SM}}$, with the Higgs mass replaced as $m_{h} \rightarrow \sqrt{s}$. This leads to the cross section

$$
\sigma\left(N_{\mathrm{DM}} N_{\mathrm{DM}} \rightarrow X_{\mathrm{SM}} \bar{X}_{\mathrm{SM}}\right) v_{r}=8 \sqrt{s} s_{h}^{2} c_{h}^{2} y_{1}^{2}\left|\frac{1}{s-M_{h_{1}}^{2}}-\frac{1}{s-M_{h_{2}}^{2}}\right|^{2} \Gamma_{h \rightarrow X_{\mathrm{SM}} \bar{X}_{\mathrm{SM}}}\left(m_{h} \rightarrow \sqrt{s}\right),
$$

where $\Gamma_{h \rightarrow X_{\mathrm{SM}} \bar{X}_{\mathrm{SM}}}\left(m_{h} \rightarrow \sqrt{s}\right)$ is the total decay width, with $m_{h} \rightarrow \sqrt{s}$.

Higgs channel. The DM can self-annihilate to $h_{1,2} h_{1,2}$, as seen in figure 2 -d, -e and -f. The amplitude squared is given by

$$
\begin{aligned}
|\mathcal{M}|^{2}= & 2 \tilde{y}_{\mathrm{DM}}^{2} s\left[\frac{c_{h} \lambda_{1 i k}}{s-M_{h_{1}}^{2}}+\frac{s_{h} \lambda_{2 i k}}{s-M_{h_{2}}^{2}}\right]^{2} \\
& +4 c_{i} c_{k} \tilde{y}_{\mathrm{DM}}^{3} M_{\mathrm{DM}}\left[\frac{c_{h} \lambda_{1 i k}}{s-M_{h_{1}}^{2}}+\frac{s_{h} \lambda_{2 i k}}{s-M_{h_{2}}^{2}}\right]\left(\frac{s-M_{h_{i}}^{2}+M_{h_{k}}^{2}}{t-M_{\mathrm{DM}}^{2}}+a \frac{s+M_{h_{i}}^{2}-M_{h_{k}}^{2}}{u-M_{\mathrm{DM}}^{2}}\right) \\
& +\frac{2 c_{i}^{2} c_{k}^{2} \tilde{y}_{\mathrm{DM}}^{4}}{\left(t-M_{\mathrm{DM}}^{2}\right)^{2}}\left\{4 M_{\mathrm{DM}}^{2} M_{h_{k}}^{2}+\left(M_{\mathrm{DM}}^{2}+M_{h_{i}}^{2}-t\right)\left(M_{\mathrm{DM}}^{2}+M_{h_{i}}^{2}-u\right)-s M_{h_{i}}^{2}\right\} \\
& +a^{2} \frac{2 c_{i}^{2} c_{k}^{2} \tilde{y}_{\mathrm{DM}}^{4}}{\left(u-M_{\mathrm{DM}}^{2}\right)^{2}}\left\{4 M_{\mathrm{DM}}^{2} M_{h_{i}}^{2}+\left(M_{\mathrm{DM}}^{2}+M_{h_{k}}^{2}-u\right)\left(M_{\mathrm{DM}}^{2}+M_{h_{k}}^{2}-t\right)-s M_{h_{k}}^{2}\right\} \\
& +a \frac{2 c_{i}^{2} c_{k}^{2} \tilde{y}_{\mathrm{DM}}^{4}}{\left(t-M_{\mathrm{DM}}^{2}\right)\left(u-M_{\mathrm{DM}}^{2}\right)}\left\{\left(M_{\mathrm{DM}}^{2}+M_{h_{i}}^{2}-t\right)\left(M_{\mathrm{DM}}^{2}+M_{h_{k}}^{2}-t\right)\right. \\
& \left.+\left(M_{\mathrm{DM}}^{2}+M_{h_{k}}^{2}-u\right)\left(M_{\mathrm{DM}}^{2}+M_{h_{i}}^{2}-u\right)-\left(s-4 M_{\mathrm{DM}}^{2}\right)\left(s-M_{h_{i}}^{2}-M_{h_{k}}^{2}\right)\right\}, \quad(5.7)
\end{aligned}
$$

with $s, t$ and $u$ being the Mandelstam variables, the Yukawa couplings defined as $\tilde{y}_{\mathrm{DM}} \equiv y_{1}$, $c_{1} \equiv c_{h}$ and $c_{2} \equiv s_{h}$. Here, we integrate numerically on the phase space in order to get the cross section for a given $s$ value. At tree-level the effective cubic scalar couplings $\left(\lambda_{1 i k}\right.$ and $\left.\lambda_{2 i k}\right)$ are given by

$$
\begin{aligned}
& \lambda_{111}=6 \lambda_{\mathrm{H}} c_{h}^{3} v-3 \lambda_{\phi \mathrm{H}} c_{h}^{2} s_{h} v+3 \lambda_{\phi \mathrm{H}} c_{h} s_{h}^{2} x-6 \lambda_{\phi} s_{h}^{3} x, \\
& \lambda_{112}=\lambda_{\phi \mathrm{H}} c_{h}^{3} x+2 c_{h}^{2} s_{h}\left(3 \lambda_{\mathrm{H}}-\lambda_{\phi \mathrm{H}}\right) v+2 c_{h} s_{h}^{2}\left(3 \lambda_{\phi}-\lambda_{\phi \mathrm{H}}\right) x+\lambda_{\phi \mathrm{H}} s_{h}^{3} v, \\
& \lambda_{222}=\lambda_{122}=0
\end{aligned}
$$

though for completeness we use the full one-loop results that can be derived from the loop-corrected potential following [147]. The absence of cubic interactions $h_{1} h_{2}^{2}$ and $h_{2}^{3}$, at leading order, is a general feature of SI models. 


\subsection{Direct detection}

Concerning direct-detection experiments, the effective low-energy Lagrangian responsible for interactions between the DM and quarks is given by

$$
\mathcal{L}_{N_{1}-q}^{(\mathrm{eff})}=a_{q} \bar{q} q N_{\mathrm{DM}}^{c} N_{\mathrm{DM}},
$$

with

$$
a_{q}=-\frac{s_{h} c_{h} M_{q} M_{\mathrm{DM}}}{2 v x}\left[\frac{1}{M_{h_{1}}^{2}}-\frac{1}{M_{h_{2}}^{2}}\right] .
$$

Consequently, the nucleon-DM effective interaction can be written as

$$
\mathcal{L}_{\mathrm{DM}-\mathcal{N}}^{(\mathrm{eff})}=a_{\mathcal{N}} \overline{\mathcal{N}} \mathcal{N} N_{\mathrm{DM}}^{c} N_{\mathrm{DM}},
$$

with

$$
a_{\mathcal{N}}=\frac{s_{h} c_{h}\left(M_{\mathcal{N}}-\frac{7}{9} M_{\mathcal{B}}\right) M_{\mathrm{DM}}}{v x}\left[\frac{1}{M_{h_{1}}^{2}}-\frac{1}{M_{h_{2}}^{2}}\right] .
$$

In this relation, $M_{\mathcal{N}}$ is the nucleon mass and $M_{\mathcal{B}}$ the baryon mass in the chiral limit [148]. Thus, the approximate expression of the spin-independent nucleon-DM elastic cross section at low momentum transfer reads

$$
\sigma_{\mathrm{det}}=\frac{s_{h}^{2} c_{h}^{2} M_{\mathcal{N}}^{2}\left(M_{\mathcal{N}}-\frac{7}{9} M_{\mathcal{B}}\right)^{2} M_{\mathrm{DM}}^{4}}{\pi v^{2} x^{2}\left(M_{\mathrm{DM}}+M_{\mathcal{B}}\right)^{2}}\left[\frac{1}{M_{h_{1}}^{2}}-\frac{1}{M_{h_{2}}^{2}}\right]^{2} .
$$

As will be discussed below, the most stringent constraint on $\sigma_{\text {det }}$ comes from the present as well the recent upped bound reported by LUX experiment $[149,150]$.

\section{$6 \quad$ Numerical analysis and results}

In our numerical scan we enforce the minimization conditions, eqs. (2.7) and (2.8), vacuum stability, the Higgs mass $M_{h_{2}}=125.09 \mp 0.21 \mathrm{GeV}$, as well as the constraints from LEP (OPAL) on a light Higgs [151]. The constraint from the Higgs invisible decay $\mathcal{B}(h \rightarrow$ inv $)<$ $17 \%$ [140] is also enforced. All dimensionless couplings are restricted to perturbative values and we consider the range $200 \mathrm{GeV}<\langle\phi\rangle<5 \mathrm{TeV}$ for the beyond-SM VEV. We find a range of viable values for $M_{h_{2}}$, consistent with the OPAL bounds, as shown in figure 3. For the parameter space in our scan we tend to find $M_{h_{2}}$ in the range $\mathcal{O}(1) \mathrm{GeV} \lesssim M_{h_{2}} \lesssim 90 \mathrm{GeV}$. Lighter values of $M_{h_{2}}$ appear to require a degree of engineered cancellation among the radiative mass-corrections from fermions and bosons; see eq. (2.17). We noticed that regions with $\langle\phi\rangle \gtrsim 700 \mathrm{GeV}$ tend to be preferred in our scans.

We also scan for viable neutrino masses and mixing, subject to the LFV and muon anomalous magnetic moment constraints, while also demanding a viable DM relic density. In figure 4 we plot viable benchmark points for the Yukawa couplings $g_{i \alpha}$ and $f_{\alpha \beta}$, along with the corresponding LFV branching ratios and $\delta a_{\mu}$ contributions. It is clear that the couplings $f_{\alpha \beta}$ are generally smaller than the couplings $g_{i \alpha}$, and that the bound on $\tau \rightarrow \mu \gamma$ is readily satisfied, while the constraint from $\mu \rightarrow e \gamma$ is more severe. We observe in figure 4 


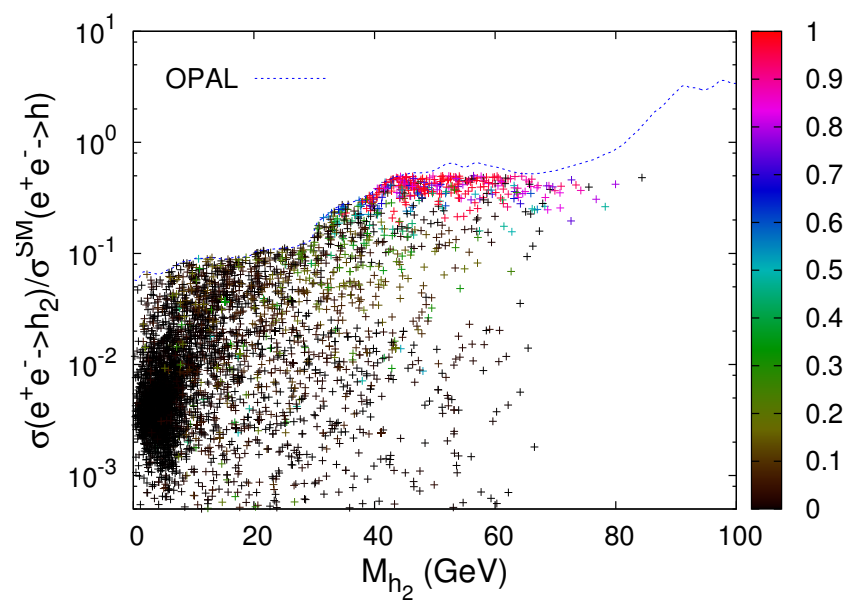

Figure 3. Scalar mixing versus the light scalar mass. The palette gives the branching ratio for invisible Higgs decays, with an overwhelming majority of the points shown satisfying the constraint $B\left(h_{1} \rightarrow\right.$ inv $)<17 \%$.

that the model requires the largest coupling in the set $g_{i \alpha}$ to take $\mathcal{O}(1)$ values. This feature is a generic expectation for three-loop models of neutrino mass, as one cannot make the new physics arbitrarily heavy, while reducing the Yukawa couplings, and retain viable SM neutrino masses. Thus, the testability of such models, which predict new physics at the $\mathrm{TeV}$ scale, is generally coupled with a need for $\mathcal{O}(1)$ couplings. Consequently one expects such couplings to encounter a Landau pole in the UV, requiring a new description. We note that, when considering only one or two generations of singlet fermions, no solutions that simultaneously accommodate the neutrino mass and mixing data, low-energy flavor constraints, and the DM relic density, were found. Therefore at least three generations of exotic fermions are required. Also, we verified that the constraints from neutrino-less double-beta decay searches are easily satisfied for all benchmark points.

Recall that, with regards to the DM relic density, there are many classes of annihilation channels, namely $N_{\mathrm{DM}} N_{\mathrm{DM}} \rightarrow X\left(X=l_{\alpha}^{-} l_{\beta}^{+}, b \bar{b}, t \bar{t}, W W+Z Z, h_{1,2} h_{1,2}\right)$. According to the DM mass, each channel could be significant or suppressed. In order to probe the role of each channel, we plot the relative contribution of each channel to the total cross section, i.e. the ratio $\sigma_{X} / \sigma_{\text {tot }}$ at the freeze-out versus the DM mass, in figure 5-left. We see that the channel $N_{\mathrm{DM}} N_{\mathrm{DM}} \rightarrow l_{\alpha}^{-} l_{\beta}^{+}$is always fully dominant except for a few benchmark points. For DM masses smaller than $80 \mathrm{GeV}$ the contribution of $X=b \bar{b}$ can be significant, while in the range between $80 \mathrm{GeV}<M_{\mathrm{DM}}<100 \mathrm{GeV}$, both gauge bosons $X=W W+Z Z$ and $X=t \bar{t}$ contributions can be important. In the range $200 \mathrm{GeV}<M_{\mathrm{DM}}<400 \mathrm{GeV}$, their contribution can reach $20 \%$. For large DM masses $M_{\mathrm{DM}}>200 \mathrm{GeV}$, the $X=h h$ contribution can reach at most $8 \%$. The fact that the $X=t \bar{t}$ contribution could be important around $100 \mathrm{GeV}$, i.e., for $M_{\mathrm{DM}}<M_{t}$, can be understood due to thermal fluctuations. Figure 5-right shows the corresponding charged scalar masses. For lighter DM masses of $M_{\text {DM }}<300 \mathrm{GeV}$, the charged scalar masses $M_{S_{1,2}}$ should not exceed $450 \mathrm{GeV}$, while for larger values of $M_{\mathrm{DM}}$, the scalar masses $M_{S_{1,2}}$ can be at the TeV scale. Such light charged scalars can be within reach of collider experiments [152]. 

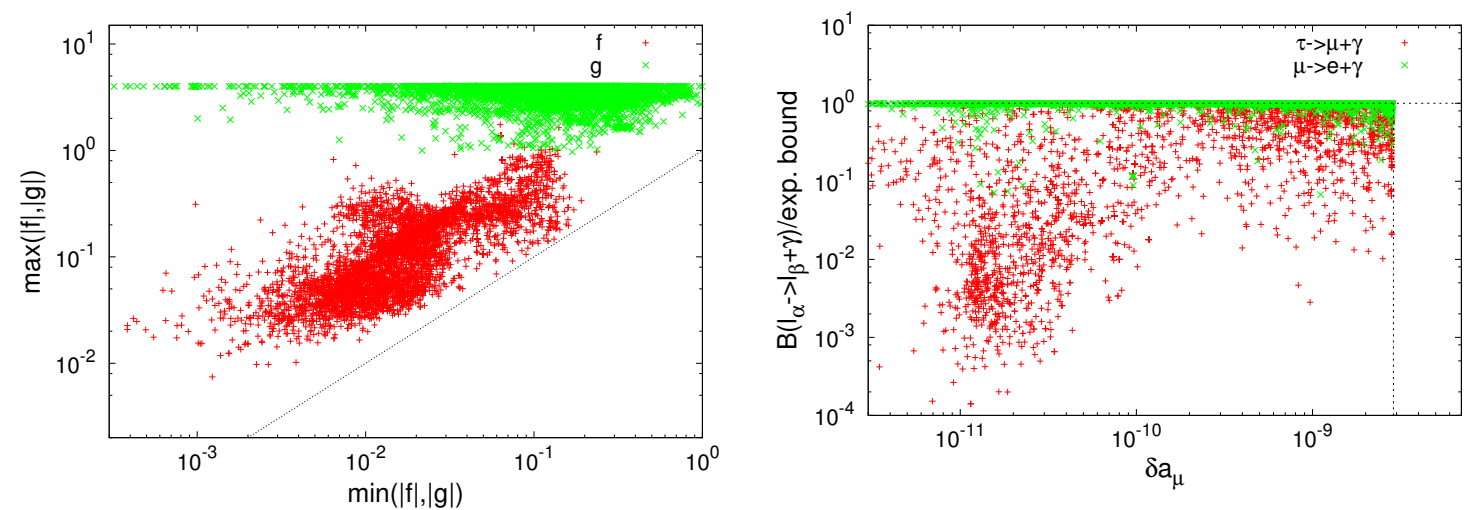

Figure 4. Left: viable benchmark points for the Yukawa couplings $g_{i \alpha}$ and $f_{\alpha \beta}$, in absolute values, where the dashed line represents the fully degenerate case, i.e, $\min |f|=\max |f|$. Right: the LFV branching ratios, scaled by the experimental bounds, versus the muon anomalous magnetic moment. The vertical line represents the muon anomalous magnetic moment experimental constraint.
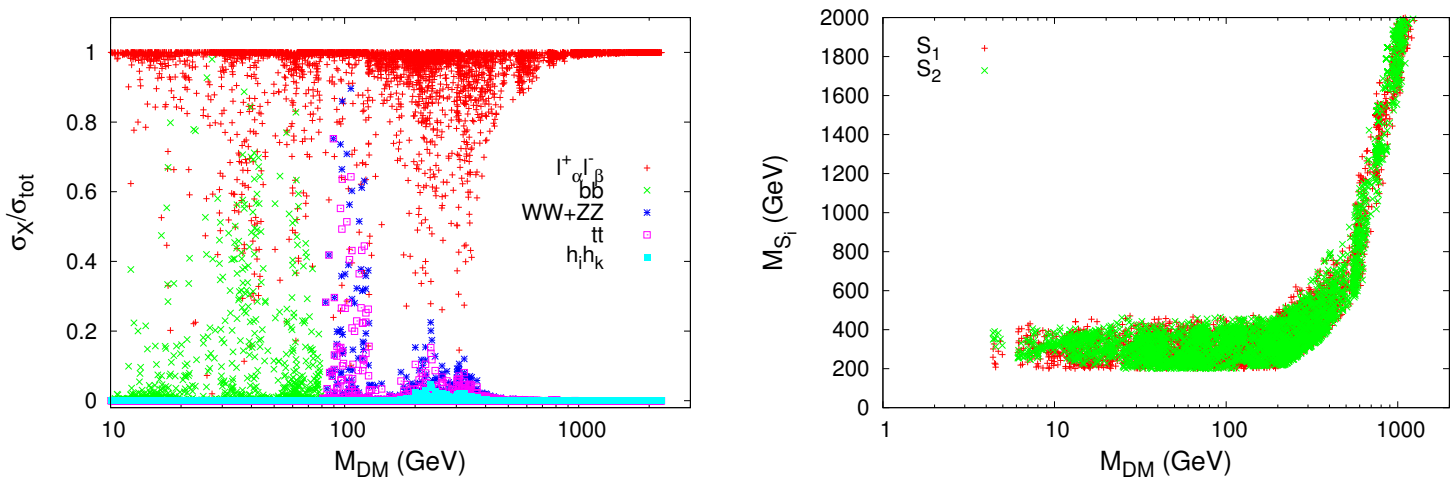

Figure 5. Left: the relative contributions of each channel to the annihilation cross section at the freeze-out temperature versus the DM mass. Right: the corresponding charged scalar masses versus the DM mass.

Next we discuss the constraints from direct-detection experiments. We plot the directdetection cross section versus the DM mass for our benchmark points in figure 6 . One observes immediately that the direct-detection limits impose serious constraints on the model, with a large number of the benchmarks excluded by LUX [149] as well the improved LUX bounds [150]. We find that only few benchmarks with $M_{\mathrm{DM}} \lesssim 10 \mathrm{GeV}$ or $M_{\mathrm{DM}} \gtrsim$ $400 \mathrm{GeV}$ survive the LUX bounds. As is clear from the figure, the surviving benchmarks will be subject to future tests in forthcoming direct-detection experiments. The palette in figure 6 shows the corresponding values for $M_{h_{2}}$, in units of $\mathrm{GeV}$. In the region of parameter space for which $N_{\mathrm{DM}}$ gives viable dark matter, we find that the $M_{h_{2}}$ must be greater than $20 \mathrm{GeV}$.

We emphasize that we only found a few benchmarks for which the DM relic density was primarily determined by annihilations into scalars. On the surface, this claim may appear contrary to the results of refs. [153, 154], which consider Majorana DM coupled to 


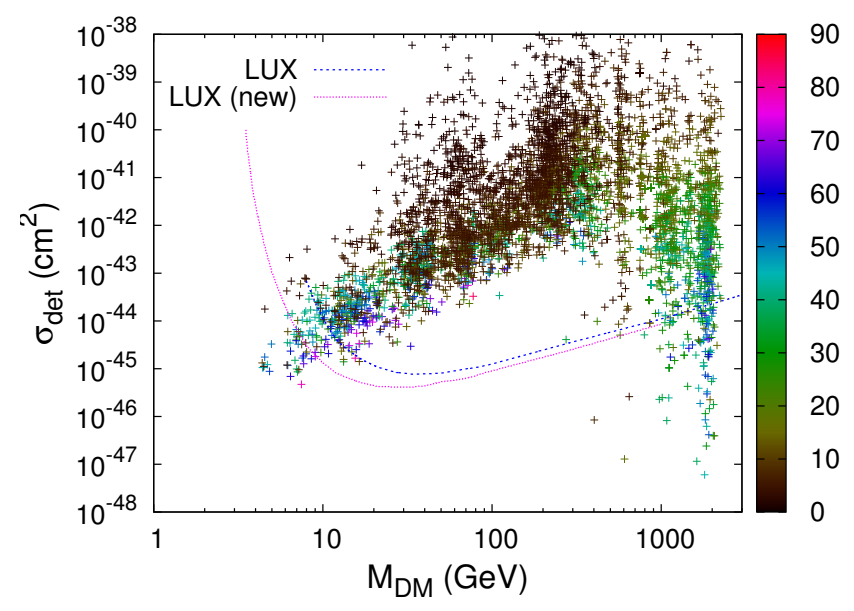

Figure 6. The direct detection cross section versus the DM mass compared to the recent results from LUX. The palette shows the mass for the neutral beyond-SM scalar, $M_{h_{2}}$, in units of GeV.

a singlet scalar that communicates with the SM via the Higgs portal (called the Indirect Higgs Portal [153]). Naively one may expect our model to admit parameter space where the DM relic-density is determined primarily by the annihilations $N_{\mathrm{DM}} N_{\mathrm{DM}} \rightarrow h h$, in analogy with the results of refs. $[153,154]$. However, due to the SI symmetry, our model contains no bare mass terms, which reduces the number of free parameters in the Lagrangian. Consequently the DM mass $M_{\mathrm{DM}}$ is related to both the coupling between $N_{\mathrm{DM}}$ and $\phi$, and the mixing angle $\theta_{h}$. This reduction in parameters means we cannot evade the LUX constraints whilst generating a viable relic density by annihilations into scalars, explaining the difference between our results and refs. $[153,154]$. It also explains some features of the benchmark distributions in figure 6 . The benchmarks with larger contributions from the channel $N_{\mathrm{DM}} N_{\mathrm{DM}} \rightarrow h h$ have a stronger coupling between $N_{\mathrm{DM}}$ and $\phi$. This increases the direct-detection cross section due to $h_{1,2}$ exchange, creating conflict with the bounds from LUX, so the corresponding benchmarks are strongly ruled out. Indeed, with the smaller number of parameters in the SI model, it is a non-trivial result that viable regions of parameter space were found in figure 6 .

Finally, we mention that the exotics in the model allow for new contributions to the Higgs decays $h \rightarrow \gamma \gamma$ and $h \rightarrow \gamma Z$. We plot the ratio of the corresponding widths relative to the SM values in figure 7-right. We observe that a significant portion of the benchmarks are consistent with existing constraints from ATLAS and CMS. Importantly, the model can be probed through more precise measurements by ATLAS and CMS after Run II. We note that all benchmark points are consistent with the oblique parameter constraints, as shown in figure 7-left.

\section{Conclusion}

We presented a scale-invariant extension of the SM in which both the weak scale and neutrino mass were generated radiatively. The model contains a DM candidate, in the form of a sterile neutrino $N_{\mathrm{DM}}$. A new light neutral scalar is also predicted, namely the pseudo 

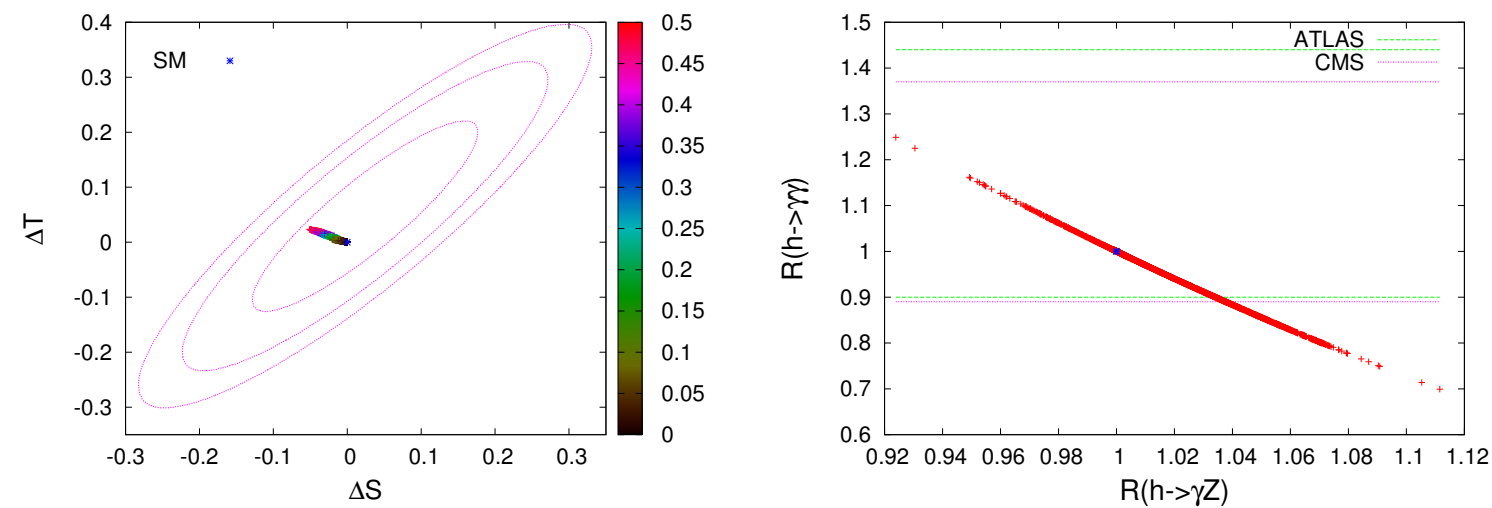

Figure 7. Left: the oblique parameters $\Delta S$ versus $\Delta T$ for the benchmarks used previously. The palette shows the mixing $\sin ^{2} \theta_{h}$ and all the points are inside the ellipsoid of $68 \%$ CL. Right: ratio of the widths for $h \rightarrow \gamma \gamma$ and $h \rightarrow \gamma Z$ relative to the SM values. The constraints from ATLAS and CMS are shown, along with projected sensitivities after Run II at the LHC.

Goldstone-boson associated with the broken scale-invariance, $h_{2}$, along with two charged scalars $S_{1,2}$. The masses for the latter are generically expected to be near the TeV scale, due to the related birth of the exotic scale and the weak scale via dimensional transmutation. The constraints on the model are rather strong, particularly the direct-detections constraints from LUX. However, we demonstrated the existence of viable parameter space with $M_{\mathrm{DM}} \lesssim 10 \mathrm{GeV}$ or $M_{\mathrm{DM}} \gtrsim 400 \mathrm{GeV}$. The model can be tested in a number of ways, including future direct-detection experiments, collider searches for the charged scalars, improved LFV searches, and precision measurements of the Higgs decay width to neutral gauge bosons. We note that the model does not possess an obvious mechanism for baryogenesis - it would be interesting to study this matter further. In a partner paper we shall study the scale-invariant implementation of the Ma model in refs. [4-16].

\section{Acknowledgments}

AA wants to thank the ICTP for the hospitality during the last stage of this work. AA is supported by the Algerian Ministry of Higher Education and Scientific Research under the CNEPRU Project No D01720130042. KM is supported by the Australian Research Council.

\section{A Multi-scalar scale-invariant theories}

In a general multi-scalar theory one cannot minimize the full one-loop corrected potential analytically. However, with recourse to the underlying SI symmetry, there exists a simple analytic approximation that captures the leading features [126]. A general tree-level SI potential for a set of scalars $\left\{\phi_{A}\right\}$ can be written as

$$
V_{0}\left(\left\{\phi_{A}\right\}\right)=g_{A B C D} \phi_{A} \phi_{B} \phi_{C} \phi_{D}
$$


where the dimensionless couplings $g_{A B C D}$ are symmetric. In general, these couplings are running parameters that depend on the energy scale, $g_{A B C D}=g_{A B C D}(\mu)$, and one can freely select a value of $\mu$ that simplifies the analysis. A convenient choice is the value $\mu=\Lambda$, at which the tree-level potential vanishes along the direction of an assumed nontrivial minimum in field space, namely

$$
g_{A B C D}(\Lambda) \hat{\phi}_{A} \hat{\phi}_{B} \hat{\phi}_{C} \hat{\phi}_{D}=0
$$

Here, the minimum is defined by $\left\langle\phi_{A}\right\rangle=R \hat{\phi}_{A}$, with $\hat{\phi}_{A}$ a unit vector in field space and $R$ a (yet to be determined) radius. Combining eq. (A.2) with the minimization conditions, $\partial V_{0} / \partial \phi_{A}=0$, determines the angular VEVs $\hat{\phi}_{A}$ in terms of the couplings $g_{A B C D}$. Subsequently expanding around the ground state in the tree-level potential reveals a spectrum containing a massless scalar, corresponding to the flat direction.

Eq. (A.2) implies that the tree-level potential vanishes, at the scale $\mu=\Lambda$, to an accuracy on the order of the loop corrections:

$$
V_{0}\left(\left\{R \hat{\phi}_{A}\right\} ; \mu=\Lambda\right) \lesssim \mathcal{O}\left(V_{1-\text { loop }}\right)
$$

where we display the renormalization scale dependence and write the full loop-corrected potential as $V=V_{0}+V_{1-\text { loop }}+\ldots$. Thus, one-loop corrections can be comparable to $V_{0}$ along the direction $\hat{\phi}_{A}$, so the interplay of the two terms allows a non-trivial minimum that lifts the flat direction to fix the radial VEV $\langle R\rangle$. Adding the one-loop corrections along the direction $\hat{\phi}_{A}$ gives

$$
V\left(\left\{R \hat{\phi}_{A}\right\} ; \mu=\Lambda\right)=V_{0}\left(\left\{R \hat{\phi}_{A}\right\} ; \mu=\Lambda\right)+V_{1-\operatorname{loop}}\left(\left\{R \hat{\phi}_{A}\right\} ; \mu=\Lambda\right)+\ldots,
$$

which can be written as [126]

$$
V\left(\left\{R \hat{\phi}_{A}\right\} ; \mu=\Lambda\right)=\mathcal{A} R^{4}+\mathcal{B} R^{4} \log \frac{R^{2}}{\Lambda^{2}}+\ldots,
$$

with

$$
\mathcal{A}=\frac{1}{64 \pi^{2}\langle R\rangle^{4}}\left\{\operatorname{Tr}\left[\mathcal{M}_{S}^{4} \log \frac{\mathcal{M}_{S}^{2}}{\langle R\rangle^{2}}\right]-\operatorname{Tr}\left[\mathcal{M}_{F}^{4} \log \frac{\mathcal{M}_{F}^{2}}{\langle R\rangle^{2}}\right]+\operatorname{Tr}\left[\mathcal{M}_{V}^{4} \log \frac{\mathcal{M}_{V}^{2}}{\langle R\rangle^{2}}\right]\right\}
$$

and

$$
\mathcal{B}=\frac{1}{64 \pi^{2}\langle R\rangle^{4}}\left\{\operatorname{Tr} \mathcal{M}_{\mathrm{S}}^{4}-\operatorname{Tr} \mathcal{M}_{\mathrm{F}}^{4}+\operatorname{Tr} \mathcal{M}_{\mathrm{V}}^{4}\right\}
$$

Here $\mathcal{M}_{\mathrm{S}, \mathrm{F}, \mathrm{V}}$ are the mass matrices for scalars, fermions and vectors, respectively, and the trace runs over both particle species and internal degrees of freedom. Minimizing the one-loop corrected potential lifts the flat-direction to give

$$
\langle R\rangle=e^{-\{\mathcal{A} / 2 \mathcal{B}+1 / 4\}} \Lambda .
$$

The dilaton acquires a loop-level mass, given by $M_{\text {dilaton }}^{2}=8 \mathcal{B}\langle R\rangle^{2}$. Thus, radiative corrections successfully induce a non-trivial VEV for one or more of the scalars $\phi_{A}$, by introducing 
a dimensionful parameter, $\langle R\rangle \propto \Lambda$, in exchange for one of the dimensionless couplings in eq. (A.2). This manifests dimensional transmutation.

In the present model, demanding that $M_{\text {dilaton }}^{2}=8 \mathcal{B}\langle R\rangle^{2}>0$, requires that $\mathcal{B}$ be dominated by the term $\operatorname{Tr} \mathcal{M}_{\mathrm{S}}^{4}$, meaning that one (or both) of the scalars $S_{1,2}^{+}$must be the heaviest state in the spectrum. In practise, this implies that $\mathcal{A}$ is also dominated by the contribution of $S_{1,2}^{+}$to the $\mathcal{M}_{\mathrm{S}}^{4}$ term in eq. (A.6). Thus, loop corrections from the scalars $h_{1,2}$ along the flat direction are sub-dominant to the corrections from $S_{1,2}^{+} \cdot{ }^{8}$ Therefore, simply dropping the corrections from $h_{1,2}$ will not introduce a significant error in the analysis (the error is expected to be $\mathcal{O}\left(M_{h_{1}}^{4} / M_{S_{1,2}}^{4}\right)$ ). As discussed in the text, this simplification has the advantage of allowing one to obtain analytic expressions for the ground state by minimizing the one-loop corrected potential directly. As a point of comparison, for the present model, the minimization in eq. (A.2) gives $4 \sqrt{\lambda_{\mathrm{H}}(\Lambda) \lambda_{\phi}(\Lambda)}+\lambda_{\phi \mathrm{H}}(\Lambda)=0$, and we see from eq. (2.7) that our approach incorporates loop corrections to this expression, up to $\mathcal{O}\left(M_{h_{1}}^{4} / M_{S_{1,2}}^{4}\right)$ effects. Taking the heaviest scalar as $M_{S} \gtrsim 300 \mathrm{GeV}$ (which we can always do - see figure 5 ), the error in the loop terms is typically $\lesssim 3 \%$. Once we have found the ground state, we reintroduce loop corrections from $h_{1,2}$ to determine the mass eigenvalues, reducing the error in the expressions for the scalar masses and mixings.

\section{B Oblique parameter functions}

The functions employed in the calculation of the oblique parameters in section 4 are defined as follows:

$$
\begin{aligned}
F(I, J) \equiv & \begin{array}{l}
\frac{I+J}{2}-\frac{I J}{I-J} \ln \frac{I}{J} \Leftarrow I \neq J \\
0 \quad \Leftarrow I=J
\end{array} \\
G(I, J, Q) \equiv & -\frac{16}{3}+\frac{5(I+J)}{Q}-\frac{2(I-J)^{2}}{Q^{2}} \\
& +\frac{3}{Q}\left[\frac{I^{2}+J^{2}}{I-J}-\frac{I^{2}-J^{2}}{Q}+\frac{(I-J)^{3}}{3 Q^{2}}\right] \ln \frac{I}{J}+\frac{r}{Q^{3}} f(t, r), \\
\hat{G}(I, Q)= & -\frac{79}{3}+9 \frac{I}{Q}-2 \frac{I^{2}}{Q^{2}}+\left(-10+18 \frac{I}{Q}-6 \frac{I^{2}}{Q^{2}}+\frac{I^{3}}{Q^{3}}-9 \frac{I+Q}{I-Q}\right) \ln \frac{I}{Q} \\
& +\left(12-4 \frac{I}{Q}+\frac{I^{2}}{Q^{2}}\right) \frac{f\left(I, I^{2}-4 I Q\right)}{Q},
\end{aligned}
$$

with $t \equiv I+J-Q$ and $r \equiv Q^{2}-2 Q(I+J)+(I-J)^{2}$, and

$$
f(t, r) \equiv\left\{\begin{aligned}
\sqrt{r} \ln \left|\frac{t-\sqrt{r}}{t+\sqrt{r}}\right| & \Leftarrow r>0, \\
0 & \Leftarrow r=0, \\
2 \sqrt{-r} \arctan \frac{\sqrt{-r}}{t} & \Leftarrow r<0 .
\end{aligned}\right.
$$

\footnotetext{
${ }^{8}$ For parameter space of interest in this work, corrections from $h_{1,2}$ are also smaller than those from the top quark and, in large regions of parameter space, one or more of the fermions $N$.
} 


\section{Loop induced Higgs decay functions}

The functions used to evaluate the Higgs decay rate of $h \rightarrow \gamma \gamma$ are given by

$$
\begin{aligned}
A_{0}^{\gamma \gamma}(x) & =-x^{-2}[x-f(x)], \\
A_{1 / 2}^{\gamma \gamma}(x) & =2 x^{-2}[x+(x-1) f(x)], \\
A_{1}^{\gamma \gamma}(x) & =-x^{-2}\left[2 x^{2}+3 x+3(2 x-1) f(x)\right],
\end{aligned}
$$

with

$$
f(x)=\left\{\begin{array}{cc}
\arcsin ^{2}(\sqrt{x}) & x \leq 1 \\
-\frac{1}{4}\left[\log \frac{1+\sqrt{1-x^{-1}}}{1-\sqrt{1-x^{-1}}}-i \pi\right]^{2} & x>1
\end{array}\right.
$$

and those used in the decay rate of $h \rightarrow \gamma Z$ are given by

$$
\begin{aligned}
& A_{0}^{\gamma Z}(x, y)=I_{1}(x, y), \\
& A_{1 / 2}^{\gamma Z}(x, y)=I_{1}(x, y)-I_{2}(x, y), \\
& A_{1}^{\gamma Z}(x, y)=\left[(1+2 x) \tan ^{2} \theta_{w}-(5+2 x)\right] I_{1}(x, y)+4\left(3-\tan ^{2} \theta_{w}\right) I_{2}(x, y),
\end{aligned}
$$

with

$$
I_{1}(x, y)=-\frac{1}{2(x-y)}+\frac{f(x)-f(y)}{2(x-y)^{2}}+\frac{y[g(x)-g(y)]}{(x-y)^{2}}, I_{2}(x, y)=\frac{f(x)-f(y)}{2(x-y)},
$$

and

$$
g(x)=\left\{\begin{array}{cc}
\sqrt{x^{-1}-1} \arcsin (\sqrt{x}) & x \leq 1 \\
\frac{\sqrt{1-x^{-1}}}{2}\left[\log \frac{1+\sqrt{1-x^{-1}}}{1-\sqrt{1-x^{-1}}}-i \pi\right] & x>1 .
\end{array}\right.
$$

Open Access. This article is distributed under the terms of the Creative Commons Attribution License (CC-BY 4.0), which permits any use, distribution and reproduction in any medium, provided the original author(s) and source are credited.

\section{References}

[1] S.R. Coleman and E.J. Weinberg, Radiative Corrections as the Origin of Spontaneous Symmetry Breaking, Phys. Rev. D 7 (1973) 1888 [INSPIRE].

[2] A. Kobakhidze and K.L. McDonald, Comments on the Hierarchy Problem in Effective Theories, JHEP 07 (2014) 155 [arXiv: 1404.5823] [InSPIRE].

[3] L.M. Krauss, S. Nasri and M. Trodden, A model for neutrino masses and dark matter, Phys. Rev. D 67 (2003) 085002 [hep-ph/0210389] [INSPIRE].

[4] E. Ma, Verifiable radiative seesaw mechanism of neutrino mass and dark matter, Phys. Rev. D 73 (2006) 077301 [hep-ph/0601225] [INSPIRE].

[5] M. Aoki, S. Kanemura and O. Seto, Neutrino mass, Dark Matter and Baryon Asymmetry via TeV-Scale Physics without Fine-Tuning, Phys. Rev. Lett. 102 (2009) 051805 [arXiv:0807.0361] [INSPIRE].

[6] M. Aoki, S. Kanemura and O. Seto, A Model of TeV Scale Physics for Neutrino Mass, Dark Matter and Baryon Asymmetry and its Phenomenology, Phys. Rev. D 80 (2009) 033007 [arXiv: 0904.3829] [INSPIRE]. 
[7] M. Aoki, S. Kanemura, T. Shindou and K. Yagyu, An R-parity conserving radiative neutrino mass model without right-handed neutrinos, JHEP 07 (2010) 084 [Erratum ibid. 1011 (2010) 049] [arXiv:1005.5159] [INSPIRE].

[8] S. Kanemura, O. Seto and T. Shimomura, Masses of dark matter and neutrino from TeV scale spontaneous $\mathrm{U}(1)_{B-L}$ breaking, Phys. Rev. D 84 (2011) 016004 [arXiv:1101.5713] [INSPIRE].

[9] M. Aoki, S. Kanemura and K. Yagyu, Doubly-charged scalar bosons from the doublet, Phys. Lett. B 702 (2011) 355 [Erratum ibid. B 706 (2012) 495] [arXiv:1105.2075] [INSPIRE].

[10] M. Lindner, D. Schmidt and T. Schwetz, Dark Matter and neutrino masses from global $\mathrm{U}(1)_{B-L}$ symmetry breaking, Phys. Lett. B 705 (2011) 324 [arXiv:1105.4626] [INSPIRE].

[11] S. Kanemura, T. Nabeshima and H. Sugiyama, TeV-Scale Seesaw with Loop-Induced Dirac Mass Term and Dark Matter from $\mathrm{U}(1)_{B-L}$ Gauge Symmetry Breaking, Phys. Rev. D 85 (2012) 033004 [arXiv:1111.0599] [INSPIRE].

[12] Y.H. Ahn and H. Okada, Non-zero $\theta_{13}$ linking to Dark Matter from Non-Abelian Discrete Flavor Model in Radiative Seesaw, Phys. Rev. D 85 (2012) 073010 [arXiv:1201.4436] [INSPIRE].

[13] S.S.C. Law and K.L. McDonald, Inverse seesaw and dark matter in models with exotic lepton triplets, Phys. Lett. B 713 (2012) 490 [arXiv:1204.2529] [INSPIRE].

[14] G. Guo, X.-G. He and G.-N. Li, Radiative Two Loop Inverse Seesaw and Dark Matter, JHEP 10 (2012) 044 [arXiv: 1207.6308] [INSPIRE].

[15] P.S. Bhupal Dev and A. Pilaftsis, Light and Superlight Sterile Neutrinos in the Minimal Radiative Inverse Seesaw Model, Phys. Rev. D 87 (2013) 053007 [arXiv:1212.3808] [INSPIRE].

[16] M. Gustafsson, J.M. No and M.A. Rivera, Predictive Model for Radiatively Induced Neutrino Masses and Mixings with Dark Matter, Phys. Rev. Lett. 110 (2013) 211802 [arXiv:1212.4806] [INSPIRE].

[17] A. Ahriche and S. Nasri, Dark matter and strong electroweak phase transition in a radiative neutrino mass model, JCAP 07 (2013) 035 [arXiv:1304.2055] [INSPIRE].

[18] A. Ahriche, C.-S. Chen, K.L. McDonald and S. Nasri, Three-loop model of neutrino mass with dark matter, Phys. Rev. D 90 (2014) 015024 [arXiv: 1404.2696] [InSPIRE].

[19] A. Ahriche, K.L. McDonald and S. Nasri, A Model of Radiative Neutrino Mass: with or without Dark Matter, JHEP 10 (2014) 167 [arXiv:1404.5917] [INSPIRE].

[20] C.-S. Chen, K.L. McDonald and S. Nasri, A Class of Three-Loop Models with Neutrino Mass and Dark Matter, Phys. Lett. B 734 (2014) 388 [arXiv:1404.6033] [INSPIRE].

[21] A. Ahriche, K.L. McDonald, S. Nasri and T. Toma, A Model of Neutrino Mass and Dark Matter with an Accidental Symmetry, Phys. Lett. B 746 (2015) 430 [arXiv:1504.05755] [INSPIRE].

[22] A. Ahriche, K.L. McDonald and S. Nasri, Scalar Sector Phenomenology of Three-Loop Radiative Neutrino Mass Models, Phys. Rev. D 92 (2015) 095020 [arXiv:1508.05881] [INSPIRE].

[23] M. Aoki, J. Kubo and H. Takano, Two-loop radiative seesaw mechanism with multicomponent dark matter explaining the possible $\gamma$ excess in the Higgs boson decay and at the Fermi LAT, Phys. Rev. D 87 (2013) 116001 [arXiv:1302.3936] [INSPIRE]. 
[24] Y. Kajiyama, H. Okada and K. Yagyu, Two Loop Radiative Seesaw Model with Inert Triplet Scalar Field, Nucl. Phys. B 874 (2013) 198 [arXiv: 1303.3463] [InSPIRE].

[25] Y. Kajiyama, H. Okada and T. Toma, Multicomponent dark matter particles in a two-loop neutrino model, Phys. Rev. D 88 (2013) 015029 [arXiv:1303.7356] [INSPIRE].

[26] S.S.C. Law and K.L. McDonald, A Class of Inert N-tuplet Models with Radiative Neutrino Mass and Dark Matter, JHEP 09 (2013) 092 [arXiv: 1305.6467] [INSPIRE].

[27] D. Restrepo, O. Zapata and C.E. Yaguna, Models with radiative neutrino masses and viable dark matter candidates, JHEP 11 (2013) 011 [arXiv:1308.3655] [INSPIRE].

[28] E. Ma, I. Picek and B. Radovčić, New Scotogenic Model of Neutrino Mass with U(1) Gauge Interaction, Phys. Lett. B 726 (2013) 744 [arXiv:1308.5313] [INSPIRE].

[29] V. Brdar, I. Picek and B. Radovcic, Radiative Neutrino Mass with Scotogenic Scalar Triplet, Phys. Lett. B 728 (2014) 198 [arXiv:1310.3183] [INSPIRE].

[30] H. Okada and K. Yagyu, Radiative generation of lepton masses, Phys. Rev. D 89 (2014) 053008 [arXiv: 1311.4360] [INSPIRE].

[31] S. Baek, H. Okada and T. Toma, Two loop neutrino model and dark matter particles with global B - L symmetry, JCAP 06 (2014) 027 [arXiv:1312.3761] [INSPIRE].

[32] S. Baek, H. Okada and T. Toma, Radiative lepton model and dark matter with global U(1)' symmetry, Phys. Lett. B 732 (2014) 85 [arXiv:1401.6921] [INSPIRE].

[33] H. Okada, Two loop Induced Dirac Neutrino Model and Dark Matters with Global U(1)' Symmetry, arXiv:1404.0280 [INSPIRE].

[34] A. Ahriche, C.-S. Chen, K.L. McDonald and S. Nasri, Three-loop model of neutrino mass with dark matter, Phys. Rev. D 90 (2014) 015024 [arXiv: 1404.2696] [InSPIRE].

[35] J.N. Ng and A. de la Puente, Probing Radiative Neutrino Mass Generation through Monotop Production, Phys. Rev. D 90 (2014) 095018 [arXiv: 1404.1415] [InSPIRE].

[36] S. Kanemura, T. Matsui and H. Sugiyama, Neutrino mass and dark matter from gauged $\mathrm{U}(1)_{B-L}$ breaking, Phys. Rev. D 90 (2014) 013001 [arXiv: 1405.1935] [INSPIRE].

[37] H. Okada and K. Yagyu, Radiative generation of lepton masses with the $\mathrm{U}(1)^{\prime}$ gauge symmetry, Phys. Rev. D 90 (2014) 035019 [arXiv:1405.2368] [INSPIRE].

[38] S. Kanemura, N. Machida and T. Shindou, Radiative neutrino mass, dark matter and electroweak baryogenesis from the supersymmetric gauge theory with confinement, Phys. Lett. B 738 (2014) 178 [arXiv:1405.5834] [INSPIRE].

[39] M. Aoki and T. Toma, Impact of semi-annihilation of $\mathbb{Z}_{3}$ symmetric dark matter with radiative neutrino masses, JCAP 09 (2014) 016 [arXiv:1405.5870] [INSPIRE].

[40] H. Ishida and H. Okada, $3.55 \mathrm{keV} \mathrm{X-ray} \mathrm{Line} \mathrm{Interpretation} \mathrm{in} \mathrm{Radiative} \mathrm{Neutrino} \mathrm{Model,}$ arXiv: 1406.5808 [INSPIRE].

[41] H. Okada and Y. Orikasa, X-ray line in Radiative Neutrino Model with Global U(1) Symmetry, Phys. Rev. D 90 (2014) 075023 [arXiv: 1407.2543] [INSPIRE].

[42] H. Okada, T. Toma and K. Yagyu, Inert Extension of the Zee-Babu Model, Phys. Rev. D 90 (2014) 095005 [arXiv:1408.0961] [INSPIRE].

[43] H. Hatanaka, K. Nishiwaki, H. Okada and Y. Orikasa, A Three-Loop Neutrino Model with Global U(1) Symmetry, Nucl. Phys. B 894 (2015) 268 [arXiv:1412.8664] [InSPIRE]. 
[44] S. Baek, H. Okada and K. Yagyu, Flavour Dependent Gauged Radiative Neutrino Mass Model, JHEP 04 (2015) 049 [arXiv: 1501.01530] [INSPIRE].

[45] L.-G. Jin, R. Tang and F. Zhang, A three-loop radiative neutrino mass model with dark matter, Phys. Lett. B 741 (2015) 163 [arXiv:1501.02020] [INSPIRE].

[46] H. Okada, Two Loop Radiative Seesaw and X-ray line Dark Matter with Global U(1) Symmetry, arXiv:1503.04557 [inSPIRE].

[47] H. Okada, N. Okada and Y. Orikasa, Radiative Seesaw in Minimal 3-3-1 Model, arXiv: 1504.01204 [INSPIRE].

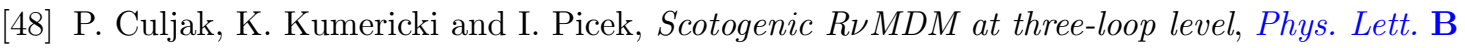
744 (2015) 237 [arXiv: 1502.07887] [InSPIRE].

[49] D. Restrepo, A. Rivera, M. Sánchez-Peláez, O. Zapata and W. Tangarife, Radiative Neutrino Masses in the Singlet-Doublet Fermion Dark Matter Model with Scalar Singlets, Phys. Rev. D 92 (2015) 013005 [arXiv: 1504.07892] [InSPIRE].

[50] S. Kashiwase, H. Okada, Y. Orikasa and T. Toma, Two Loop Neutrino Model with Dark Matter and Leptogenesis, arXiv:1505.04665 [INSPIRE].

[51] T.A. Chowdhury and S. Nasri, Lepton Flavor Violation in the Inert Scalar Model with Higher Representations, JHEP 12 (2015) 040 [arXiv:1506.00261] [INSPIRE].

[52] M. Aoki, T. Toma and A. Vicente, Non-thermal Production of Minimal Dark Matter via Right-handed Neutrino Decay, JCAP 09 (2015) 063 [arXiv: 1507.01591] [INSPIRE].

[53] K. Nishiwaki, H. Okada and Y. Orikasa, Three loop neutrino model with isolated $k^{ \pm \pm}$, Phys. Rev. D 92 (2015) 093013 [arXiv: 1507. 02412] [INSPIRE].

[54] W. Wang and Z.-L. Han, Radiative linear seesaw model, dark matter and $\mathrm{U}(1)_{B-L}$, Phys. Rev. D 92 (2015) 095001 [arXiv: 1508.00706] [INSPIRE].

[55] R. Hempfling, The Next-to-minimal Coleman-Weinberg model, Phys. Lett. B 379 (1996) 153 [hep-ph/9604278] [INSPIRE].

[56] K.A. Meissner and H. Nicolai, Conformal Symmetry and the Standard Model, Phys. Lett. B 648 (2007) 312 [hep-th/0612165] [INSPIRE].

[57] W.-F. Chang, J.N. Ng and J.M.S. Wu, Shadow Higgs from a scale-invariant hidden U(1)s model, Phys. Rev. D 75 (2007) 115016 [hep-ph/0701254] [INSPIRE].

[58] R. Foot, A. Kobakhidze and R.R. Volkas, Electroweak Higgs as a pseudo-Goldstone boson of broken scale invariance, Phys. Lett. B 655 (2007) 156 [arXiv:0704.1165] [INSPIRE].

[59] R. Foot, A. Kobakhidze, K. McDonald and R. Volkas, Neutrino mass in radiatively-broken scale-invariant models, Phys. Rev. D 76 (2007) 075014 [arXiv:0706.1829] [InSPIRE].

[60] T. Hambye and M.H.G. Tytgat, Electroweak symmetry breaking induced by dark matter, Phys. Lett. B 659 (2008) 651 [arXiv:0707.0633] [INSPIRE].

[61] R. Foot, A. Kobakhidze and R.R. Volkas, Stable mass hierarchies and dark matter from hidden sectors in the scale-invariant standard model, Phys. Rev. D 82 (2010) 035005 [arXiv: 1006.0131$]$ [INSPIRE].

[62] S. Iso, N. Okada and Y. Orikasa, Classically conformal B - L extended Standard Model, Phys. Lett. B 676 (2009) 81 [arXiv: 0902.4050] [INSPIRE]. 
[63] S. Iso, N. Okada and Y. Orikasa, The minimal B-L model naturally realized at TeV scale, Phys. Rev. D 80 (2009) 115007 [arXiv:0909.0128] [InSPIRE].

[64] M. Holthausen, M. Lindner and M.A. Schmidt, Radiative Symmetry Breaking of the Minimal Left-Right Symmetric Model, Phys. Rev. D 82 (2010) 055002 [arXiv:0911.0710] [INSPIRE].

[65] T. Hur and P. Ko, Scale invariant extension of the standard model with strongly interacting hidden sector, Phys. Rev. Lett. 106 (2011) 141802 [arXiv:1103.2571] [INSPIRE].

[66] L. Alexander-Nunneley and A. Pilaftsis, The Minimal Scale Invariant Extension of the Standard Model, JHEP 09 (2010) 021 [arXiv:1006.5916] [INSPIRE].

[67] K. Ishiwata, Dark Matter in Classically Scale-Invariant Two Singlets Standard Model, Phys. Lett. B 710 (2012) 134 [arXiv:1112.2696] [INSPIRE].

[68] J.S. Lee and A. Pilaftsis, Radiative Corrections to Scalar Masses and Mixing in a Scale Invariant Two Higgs Doublet Model, Phys. Rev. D 86 (2012) 035004 [arXiv:1201.4891] [INSPIRE].

[69] N. Okada and Y. Orikasa, Dark matter in the classically conformal B-L model, Phys. Rev. D 85 (2012) 115006 [arXiv:1202.1405] [INSPIRE].

[70] S. Iso and Y. Orikasa, TeV Scale B-L model with a flat Higgs potential at the Planck scale in view of the hierarchy problem, PTEP 2013 (2013) 023B08 [arXiv:1210.2848] [INSPIRE].

[71] C. Englert, J. Jaeckel, V.V. Khoze and M. Spannowsky, Emergence of the Electroweak Scale through the Higgs Portal, JHEP 04 (2013) 060 [arXiv:1301.4224] [INSPIRE].

[72] M. Heikinheimo, A. Racioppi, M. Raidal, C. Spethmann and K. Tuominen, Physical Naturalness and Dynamical Breaking of Classical Scale Invariance, Mod. Phys. Lett. A 29 (2014) 1450077 [arXiv: 1304.7006] [INSPIRE].

[73] T. Hambye and A. Strumia, Dynamical generation of the weak and Dark Matter scale, Phys. Rev. D 88 (2013) 055022 [arXiv: 1306.2329] [INSPIRE].

[74] I. Bars, P. Steinhardt and N. Turok, Local Conformal Symmetry in Physics and Cosmology, Phys. Rev. D 89 (2014) 043515 [arXiv: 1307.1848] [INSPIRE].

[75] M. Heikinheimo, A. Racioppi, M. Raidal and C. Spethmann, Twin Peak Higgs, Phys. Lett. B 726 (2013) 781 [arXiv:1307.7146] [INSPIRE].

[76] C.D. Carone and R. Ramos, Classical scale-invariance, the electroweak scale and vector dark matter, Phys. Rev. D 88 (2013) 055020 [arXiv:1307.8428] [INSPIRE].

[77] G. Marques Tavares, M. Schmaltz and W. Skiba, Higgs mass naturalness and scale invariance in the UV, Phys. Rev. D 89 (2014) 015009 [arXiv:1308.0025] [INSPIRE].

[78] A. Farzinnia, H.-J. He and J. Ren, Natural Electroweak Symmetry Breaking from Scale Invariant Higgs Mechanism, Phys. Lett. B 727 (2013) 141 [arXiv:1308.0295] [InSPIRE].

[79] Y. Kawamura, Naturalness, Conformal Symmetry and Duality, PTEP 2013 (2013) 113B04 [arXiv: 1308.5069] [INSPIRE].

[80] E. Gabrielli, M. Heikinheimo, K. Kannike, A. Racioppi, M. Raidal and C. Spethmann, Towards Completing the Standard Model: Vacuum Stability, EWSB and Dark Matter, Phys. Rev. D 89 (2014) 015017 [arXiv:1309.6632] [INSPIRE]. 
[81] T.G. Steele, Z.-W. Wang, D. Contreras and R.B. Mann, Viable dark matter via radiative symmetry breaking in a scalar singlet Higgs portal extension of the standard model, Phys. Rev. Lett. 112 (2014) 171602 [arXiv:1310.1960] [INSPIRE].

[82] M. Hashimoto, S. Iso and Y. Orikasa, Radiative symmetry breaking at the Fermi scale and flat potential at the Planck scale, Phys. Rev. D 89 (2014) 016019 [arXiv:1310.4304] [INSPIRE].

[83] S. Abel and A. Mariotti, Novel Higgs Potentials from Gauge Mediation of Exact Scale Breaking, Phys. Rev. D 89 (2014) 125018 [arXiv:1312.5335] [INSPIRE].

[84] C.T. Hill, Is the Higgs Boson Associated with Coleman-Weinberg Dynamical Symmetry Breaking?, Phys. Rev. D 89 (2014) 073003 [arXiv: 1401.4185] [INSPIRE].

[85] J. Guo and Z. Kang, Higgs Naturalness and Dark Matter Stability by Scale Invariance, Nucl. Phys. B 898 (2015) 415 [arXiv:1401.5609] [INSPIRE].

[86] M. Hashimoto, S. Iso and Y. Orikasa, Radiative symmetry breaking from flat potential in various U(1)' models, Phys. Rev. D 89 (2014) 056010 [arXiv:1401.5944] [INSPIRE].

[87] S. Benic and B. Radovcic, Electroweak breaking and Dark Matter from the common scale, Phys. Lett. B 732 (2014) 91 [arXiv:1401.8183] [INSPIRE].

[88] A. Salvio and A. Strumia, Agravity, JHEP 06 (2014) 080 [arXiv:1403.4226] [INSPIRE].

[89] J. Kubo, K.S. Lim and M. Lindner, Electroweak Symmetry Breaking via QCD, Phys. Rev. Lett. 113 (2014) 091604 [arXiv:1403.4262] [INSPIRE].

[90] V.V. Khoze, C. McCabe and G. Ro, Higgs vacuum stability from the dark matter portal, JHEP 08 (2014) 026 [arXiv: 1403.4953] [INSPIRE].

[91] G.C. Dorsch, S.J. Huber and J.M. No, Cosmological Signatures of a UV-Conformal Standard Model, Phys. Rev. Lett. 113 (2014) 121801 [arXiv:1403.5583] [InSPIRE].

[92] H. Davoudiasl and I.M. Lewis, Right-Handed Neutrinos as the Origin of the Electroweak Scale, Phys. Rev. D 90 (2014) 033003 [arXiv:1404.6260] [INSPIRE].

[93] J. Kubo, K.S. Lim and M. Lindner, Gamma-ray Line from Nambu-Goldstone Dark Matter in a Scale Invariant Extension of the Standard Model, JHEP 09 (2014) 016 [arXiv: 1405.1052] [INSPIRE].

[94] M. Lindner, S. Schmidt and J. Smirnov, Neutrino Masses and Conformal Electro-Weak Symmetry Breaking, JHEP 10 (2014) 177 [arXiv:1405.6204] [INSPIRE].

[95] K. Kannike, A. Racioppi and M. Raidal, Embedding inflation into the Standard Model more evidence for classical scale invariance, JHEP 06 (2014) 154 [arXiv:1405.3987] [INSPIRE].

[96] V.V. Khoze and G. Ro, Dark matter monopoles, vectors and photons, JHEP 10 (2014) 61 [arXiv: 1406.2291] [INSPIRE].

[97] D.F. Litim and F. Sannino, Asymptotic safety guaranteed, JHEP 12 (2014) 178 [arXiv: 1406.2337] [INSPIRE].

[98] G.M. Pelaggi, Predictions of a model of weak scale from dynamical breaking of scale invariance, Nucl. Phys. B 893 (2015) 443 [arXiv:1406.4104] [InSPIRE].

[99] O. Antipin, E. Mølgaard and F. Sannino, Higgs Critical Exponents and Conformal Bootstrap in Four Dimensions, JHEP 06 (2015) 030 [arXiv:1406.6166] [InSPIRE]. 
[100] W. Altmannshofer, W.A. Bardeen, M. Bauer, M. Carena and J.D. Lykken, Light Dark Matter, Naturalness and the Radiative Origin of the Electroweak Scale, JHEP 01 (2015) 032 [arXiv: 1408.3429] [INSPIRE].

[101] Y. Hamada, H. Kawai, K.-y. Oda and S.C. Park, Higgs inflation from Standard Model criticality, Phys. Rev. D 91 (2015) 053008 [arXiv:1408.4864] [INSPIRE].

[102] T.G. Steele, Z.-W. Wang and D.G.C. McKeon, Multiscale renormalization group methods for effective potentials with multiple scalar fields, Phys. Rev. D 90 (2014) 105012 [arXiv: 1409.3489] [INSPIRE].

[103] O. Antipin, M. Redi and A. Strumia, Dynamical generation of the weak and Dark Matter scales from strong interactions, JHEP 01 (2015) 157 [arXiv:1410.1817] [INSPIRE].

[104] K. Allison, C.T. Hill and G.G. Ross, An ultra-weak sector, the strong CP problem and the pseudo-Goldstone dilaton, Nucl. Phys. B 891 (2015) 613 [arXiv:1409.4029] [INSPIRE].

[105] M.B. Einhorn and D.R.T. Jones, Naturalness and Dimensional Transmutation in Classically Scale-Invariant Gravity, JHEP 03 (2015) 047 [arXiv: 1410.8513] [INSPIRE].

[106] Z. Kang, Upgrading sterile neutrino dark matter to FImP using scale invariance, Eur. Phys. J. C 75 (2015) 471 [arXiv:1411.2773] [InSPIRE].

[107] G.F. Giudice, G. Isidori, A. Salvio and A. Strumia, Softened Gravity and the Extension of the Standard Model up to Infinite Energy, JHEP 02 (2015) 137 [arXiv:1412.2769] [INSPIRE].

[108] H. Okada and Y. Orikasa, Classically Conformal Radiative Neutrino Model with Gauged B-L Symmetry, arXiv:1412.3616 [INSPIRE].

[109] Y. Hamada, H. Kawai and K.-y. Oda, Eternal Higgs inflation and the cosmological constant problem, Phys. Rev. D 92 (2015) 045009 [arXiv: 1501.04455] [INSPIRE].

[110] J. Guo, Z. Kang, P. Ko and Y. Orikasa, Accidental dark matter: Case in the scale invariant local B - L model, Phys. Rev. D 91 (2015) 115017 [arXiv:1502.00508] [INSPIRE].

[111] K. Kannike et al., Dynamically Induced Planck Scale and Inflation, JHEP 05 (2015) 065 [arXiv: 1502.01334] [INSPIRE].

[112] N.G. Nielsen, F. Sannino and O. Svendsen, Inflation from Asymptotically Safe Theories, Phys. Rev. D 91 (2015) 103521 [arXiv:1503.00702] [inSPIRE].

[113] K. Endo and Y. Sumino, A Scale-invariant Higgs Sector and Structure of the Vacuum, JHEP 05 (2015) 030 [arXiv:1503.02819] [INSPIRE].

[114] P. Humbert, M. Lindner and J. Smirnov, The Inverse Seesaw in Conformal Electro-Weak Symmetry Breaking and Phenomenological Consequences, JHEP 06 (2015) 035 [arXiv: 1503.03066] [INSPIRE].

[115] S. Oda, N. Okada and D.-s. Takahashi, Classically conformal U(1)' extended standard model and Higgs vacuum stability, Phys. Rev. D 92 (2015) 015026 [arXiv: 1504.06291] [InSPIRE].

[116] Y. Ametani, M. Aoki, H. Goto and J. Kubo, Nambu-Goldstone Dark Matter in a Scale Invariant Bright Hidden Sector, Phys. Rev. D 91 (2015) 115007 [arXiv:1505.00128] [INSPIRE].

[117] Y. Hamada, K. Kawana and K. Tsumura, Landau pole in the Standard Model with weakly interacting scalar fields, Phys. Lett. B 747 (2015) 238 [arXiv:1505.01721] [INSPIRE]. 
[118] C.D. Carone and R. Ramos, Dark chiral symmetry breaking and the origin of the electroweak scale, Phys. Lett. B 746 (2015) 424 [arXiv: 1505. 04448] [INSPIRE].

[119] Z. Kang, View FImP miracle (by scale invariance) à la self-interaction, Phys. Lett. B 751 (2015) 201 [arXiv: 1505.06554] [INSPIRE].

[120] S. Di Chiara and K. Tuominen, A minimal model for $\mathrm{SU}(N)$ vector dark matter, JHEP 11 (2015) 188 [arXiv: 1506.03285] [INSPIRE].

[121] Y. Hamada and K. Kawana, Vanishing Higgs Potential in Minimal Dark Matter Models, Phys. Lett. B 751 (2015) 164 [arXiv: 1506. 06553] [INSPIRE].

[122] J. Kubo and M. Yamada, Scale and electroweak first-order phase transitions, PTEP 2015 (2015) 093B01 [arXiv:1506.06460] [INSPIRE].

[123] K. Endo and K. Ishiwata, Direct detection of singlet dark matter in classically scale-invariant standard model, Phys. Lett. B 749 (2015) 583 [arXiv:1507.01739] [INSPIRE].

[124] A. Farzinnia, Prospects for Discovering the Higgs-like Pseudo-Nambu-Goldstone Boson of the Classical Scale Symmetry, Phys. Rev. D 92 (2015) 095012 [arXiv:1507.06926] [INSPIRE].

[125] D.M. Ghilencea, A manifestly scale-invariant regularization and quantum effective operators, arXiv:1508.00595 [INSPIRE].

[126] E. Gildener and S. Weinberg, Symmetry Breaking and Scalar Bosons, Phys. Rev. D 13 (1976) 3333 [INSPIRE].

[127] R. Foot, A. Kobakhidze, K.L. McDonald and R.R. Volkas, A solution to the hierarchy problem from an almost decoupled hidden sector within a classically scale invariant theory, Phys. Rev. D 77 (2008) 035006 [arXiv:0709.2750] [InSPIRE].

[128] R. Foot, A. Kobakhidze, K.L. McDonald and R.R. Volkas, Poincaré protection for a natural electroweak scale, Phys. Rev. D 89 (2014) 115018 [arXiv:1310.0223] [INSPIRE].

[129] B. Pontecorvo, Neutrino Experiments and the Problem of Conservation of Leptonic Charge, Sov. Phys. JETP 26 (1968) 984 [inSPIRE].

[130] Z. Maki, M. Nakagawa and S. Sakata, Remarks on the unified model of elementary particles, Prog. Theor. Phys. 28 (1962) 870 [INSPIRE].

[131] D.V. Forero, M. Tortola and J.W.F. Valle, Global status of neutrino oscillation parameters after Neutrino-2012, Phys. Rev. D 86 (2012) 073012 [arXiv:1205.4018] [INSPIRE].

[132] F. Simkovic, A. Faessler, H. Muther, V. Rodin and M. Stauf, The 0 nu bb-decay nuclear matrix elements with self-consistent short-range correlations, Phys. Rev. C 79 (2009) 055501 [arXiv: 0902.0331] [INSPIRE].

[133] B.W. Lynn, M.E. Peskin and R.G. Stuart, Radiative corrections in $\mathrm{SU}_{2} \times \mathrm{U}_{1}$, in Physics at LEP, J. Ellis and R.D. Peccei eds., CERN, Geneva (1986).

[134] D.C. Kennedy and B.W. Lynn, Electroweak Radiative Corrections with an Effective Lagrangian: Four Fermion Processes, Nucl. Phys. B 322 (1989) 1 [InSPIRE].

[135] M.E. Peskin and T. Takeuchi, A new constraint on a strongly interacting Higgs sector, Phys. Rev. Lett. 65 (1990) 964 [INSPIRE].

[136] G. Altarelli and R. Barbieri, Vacuum polarization effects of new physics on electroweak processes, Phys. Lett. B 253 (1991) 161 [INSPIRE]. 
[137] M.E. Peskin and T. Takeuchi, Estimation of oblique electroweak corrections, Phys. Rev. D 46 (1992) 381 [INSPIRE].

[138] G. Altarelli, R. Barbieri and S. Jadach, Toward a model independent analysis of electroweak data, Nucl. Phys. B 369 (1992) 3 [Erratum ibid. B 376 (1992) 444] [InSPIRE].

[139] W. Grimus, L. Lavoura, O.M. Ogreid and P. Osland, The Oblique parameters in multi-Higgs-doublet models, Nucl. Phys. B 801 (2008) 81 [arXiv:0802.4353] [INSPIRE].

[140] P. Bechtle, S. Heinemeyer, O. Stål, T. Stefaniak and G. Weiglein, Probing the Standard Model with Higgs signal rates from the Tevatron, the LHC and a future ILC, JHEP 11 (2014) 039 [arXiv: 1403.1582] [INSPIRE].

[141] A. Djouadi, The Anatomy of electro-weak symmetry breaking. I: The Higgs boson in the standard model, Phys. Rept. 457 (2008) 1 [hep-ph/0503172] [INSPIRE].

[142] G. Jungman, M. Kamionkowski and K. Griest, Supersymmetric dark matter, Phys. Rept. 267 (1996) 195 [hep-ph/9506380] [INSPIRE].

[143] G. Bertone, D. Hooper and J. Silk, Particle dark matter: Evidence, candidates and constraints, Phys. Rept. 405 (2005) 279 [hep-ph/0404175] [INSPIRE].

[144] L. Bergström, Nonbaryonic dark matter: Observational evidence and detection methods, Rept. Prog. Phys. 63 (2000) 793 [hep-ph/0002126] [InSPIRE].

[145] Planck collaboration, P.A.R. Ade et al., Planck 2015 results. XIII. Cosmological parameters, arXiv:1502.01589 [INSPIRE].

[146] K. Cheung and O. Seto, Phenomenology of TeV right-handed neutrino and the dark matter model, Phys. Rev. D 69 (2004) 113009 [hep-ph/0403003] [INSPIRE].

[147] A. Ahriche, A. Arhrib and S. Nasri, Higgs Phenomenology in the Two-Singlet Model, JHEP 02 (2014) 042 [arXiv: 1309.5615] [INSPIRE].

[148] X.-G. He, T. Li, X.-Q. Li, J. Tandean and H.-C. Tsai, Constraints on Scalar Dark Matter from Direct Experimental Searches, Phys. Rev. D 79 (2009) 023521 [arXiv:0811.0658] [INSPIRE].

[149] LUX collaboration, D.S. Akerib et al., First results from the LUX dark matter experiment at the Sanford Underground Research Facility, Phys. Rev. Lett. 112 (2014) 091303 [arXiv: 1310.8214$]$ [INSPIRE].

[150] LUX collaboration, D.S. Akerib et al., Improved WIMP scattering limits from the LUX experiment, arXiv:1512.03506 [INSPIRE].

[151] OPAL collaboration, G. Abbiendi et al., Decay mode independent searches for new scalar bosons with the OPAL detector at LEP, Eur. Phys. J. C 27 (2003) 311 [hep-ex/0206022] [INSPIRE].

[152] A. Ahriche, S. Nasri and R. Soualah, Radiative neutrino mass model at the $e^{-} e^{+}$linear collider, Phys. Rev. D 89 (2014) 095010 [arXiv:1403.5694] [INSPIRE].

[153] L. Lopez-Honorez, T. Schwetz and J. Zupan, Higgs portal, fermionic dark matter and a Standard Model like Higgs at 125 GeV, Phys. Lett. B 716 (2012) 179 [arXiv:1203.2064] [INSPIRE].

[154] M. Dutra, C.A. de S. Pires and P.S. Rodrigues da Silva, Majorana dark matter through a narrow Higgs portal, JHEP 09 (2015) 147 [arXiv: 1504.07222] [INSPIRE]. 